\title{
PRESSURE PREDICTION FOR A BLOCK OF THE JEQUITINHONHA BASIN, BRAZIL
}

\author{
Fernando T. B. Andrade, Lourenildo W. B. Leite, and Wildney W. S. Vieira
}

\begin{abstract}
Pressure prediction in sedimentary basins is relevant for mapping and/or extending a productive reservoir characterized by a low-pressure zone, which is an important principle in hydrocarbon prospecting. A major part of the efforts in this work was to structure a seismic-stratigraphic framework for modeling and imaging part of the Jequitinhonha basin, that can be used to evaluate its characteristics and potential for producing oil and gas. In practice, the main application of the method is to map a reservoir, as well as its lateral and vertical extensions. The required information for modeling is the distributions of the seismic velocities $V_{P}$ and $V_{S}$ and density $\rho$. These pieces of information can be based on velocity analysis, seismic-stratigraphic interpretations of migrated data, petrophysical data, and empirical models that can provide a relationship between velocities, density, and porosity, which can also be incorporated. Our proposed model admits vertical gravity loading as the stress-deformation generator on the geological formations, but it does not take into account the effects of curvatures, faulting, diagenesis, and lateral tectonic events as loading mechanisms. The presented robust pressure prediction method was applied to a 3D interpolated model to map the low-pressure zones (possible reservoir) capped by a high-pressure zone, as a significant petroleum geology application.
\end{abstract}

Keywords: pressure prediction, basin modeling, seismic velocity analysis.

RESUMO. A predição de pressão em bacias sedimentares é relevante para mapear e/ou estender um reservatório produtivo caracterizado por uma zona de baixa pressão, princípio importante na prospecção de hidrocarbonetos. A maior parte dos esforços deste trabalho foram em estruturar um quadro sismo-estratigráfico para a modelagem e imageamento de uma parte da bacia do Jequitinhonha, que pode ser usado para avaliar suas características e potencial para produzir óleo e gás. Na prática, a principal aplicação do método é mapear um reservatório, bem como suas extensões laterais e verticais. O conhecimento necessário para modelagem são as distribuições de velocidades $V_{P}$ e $V_{S}$ e densidade $\rho$. Estas informações podem ser baseadas na análise de velocidade, em interpretações sismo-estratigráficas de dado migrado, dados petrofísicos e modelos empíricos que podem fornecer uma relação entre velocidades, densidade e porosidade, os quais também podem ser incorporados. Nosso modelo admite a carga gravitacional vertical como gerador de tensão-deformação nas formações geológicas, mas ele não leva em consideração os efeitos de curvaturas, falhamentos, diagênese e eventos tectônicos laterais como carga forçante. O método robusto de predição de pressão apresentado foi aplicado a um modelo 3D interpolado, objetivando mapear zonas de baixa pressão (possível reservatório) capeadas por uma zona de alta pressão, como uma significativa aplicação na geologia do petróleo.

Palavras-chave: predição de pressão, modelagem de bacia sedimentar, análise de velocidade sísmica. 


\section{INTRODUCTION}

This paper describes a numerical experiment aimed at basin modeling and pressure prediction, where the main purpose is to map zones of low- and high-pressure that act as natural pumps for fluid accumulation in sedimentary basins. For this purpose, we use six seismic lines of the Jequitinhonha basin, located to the east of the state of Bahia (Brazil), the marine side (see Fig. 1). The lines used were separated into two groups: three lines in the NE-SW (L214-266, L214-268, L214270) and three lines in the NW-SE direction (L214-297, L214-302, L214-303), to build what we call an empirical 3D model.

In principle, we need a 3D survey in order to construct a consistent subsurface distribution of velocities $\left(V_{P}\right.$ and $\left.V_{S}\right)$ and density $(\rho)$. Since the surveyed lines are independent, then we need to interpolate the results obtained for each time section to build a limited block, but that can offer plausible geological information. A precursor to the present paper describing the basic and fundamental theory can be followed in Vieira et al. (2017).

The methodology is composed of a sequence of processing techniques starting with the CRS stack and migration (Mann , 2002; Cohen \& Stockwell , 2005), interpretation of reflectors (interfaces), time-depth conversion, and construction of a controlled empirical model for P- and S-wave velocities and density, necessary for prediction of subsurface pressure; that is, to map zones of low- and high-pressure. We classify the pressure prediction modeling as a post-stack migration seismic method.

In this direction, Sibiryakov et al. (2014) presented the basic theory and Andrade F.T.B. (2018) detailed results on which the present work was based. Pressure prediction, in sedimentary basin modeling for oil and gas exploration, is certainly a very attractive subject for giving a complex geology a complementary understanding of the location of a borehole for a productive well.

Seismic interpretation of the sections consisted of recognizing textures existing between the main reflective patterns. For this step, four basic principles were considered: resolution (vertical), continuity (horizontal), texture (of the intervals between dominant events), and frequency content (low, medium, high) Payton (1977).

According to Mello et al. (1994), the Regência Petroleum System is responsible for all hydrocarbon occurrences in the Jequitinhonha Basin. Furthermore, we propose that a rock is a reservoir candidate if it fulfills the geomechanical condition of a low-pressure zone capped by a high-pressure zone, and not only based on pure geological criteria. This condition is what we would like to explore in this work.

The modeling of a basin and petroleum system aiming at hydrocarbon exploration involves several theoretical aspects related to geology, geochemistry, geophysics, and engineering, as described classically by
Hantschel \& Kauerauf (2009). But, in this paper, we apply a specific data-driven method based on the distributions of $V_{\mathrm{P}}(\mathbf{x}), V_{\mathrm{S}}(\mathbf{x})$, and $\rho(\mathbf{x})$ as a priori knowledge to map low-pressure zones in a 3D basin modeling, considered important to locate a successful drilling area for oil and gas exploration.

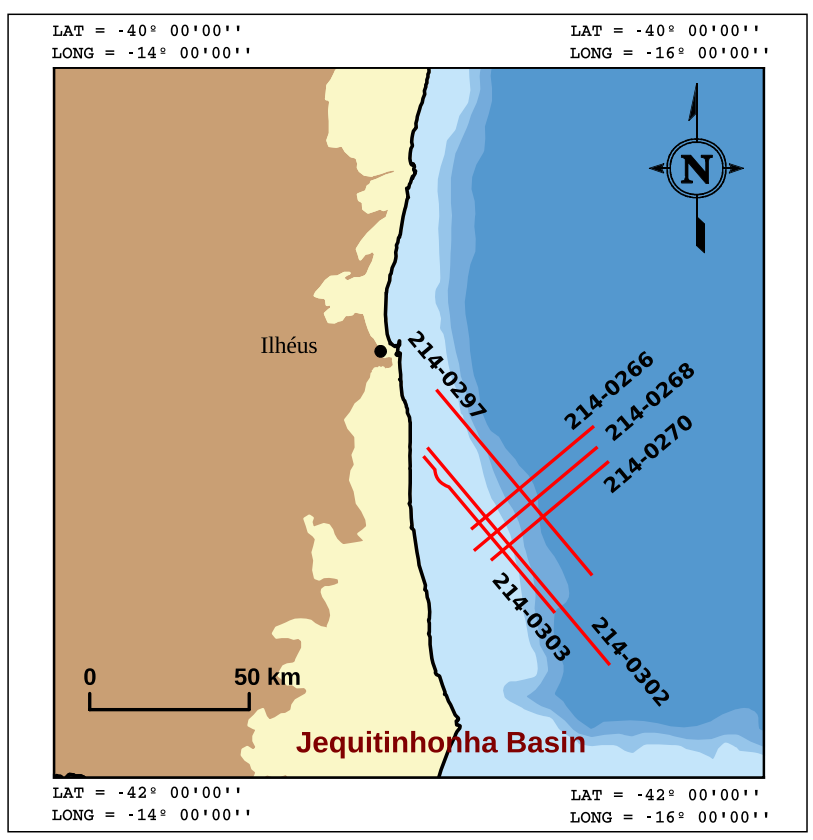

Figure 1. Location of the six seismic lines of the Jequitinhonha basin used in this work.

\section{METHODOLOGY}

The seismic data under study are presented in Figures 2 and 3 , which show the interpreted zero-offset stack sections with the common reflection surface (CRS) technology. The macro geometrical interpretation was based on reflections with clear lateral continuity of the reflector and on the texture of the macro intervals, where the sequences and structures are indicated by letters and numbers. Interpretation outlines basement highs, the layered sequence displaying lateral variations, and specific textures. The flat top of the figures shows clearly the contact water/sediments with similar behaviors of a flatter platform followed by the marine slope.

\section{Mechanics of Solids}

The main physical parameter governing pressure prediction is the measure of discontinuity in the $\gamma=\frac{V_{S}}{V_{P}}$ ratio across the geological interfaces, where $V_{S}$ is the Swave velocity and $V_{P}$ is the P-wave velocity. Sibiryakov et al. (2015) deal with different aspects of this special method, where it is described that, as a result of the $\gamma$ ratio behavior, an anticline is not necessarily the only structural condition for potentiating a zone to be an oil and gas accumulator (reservoir, collector). 

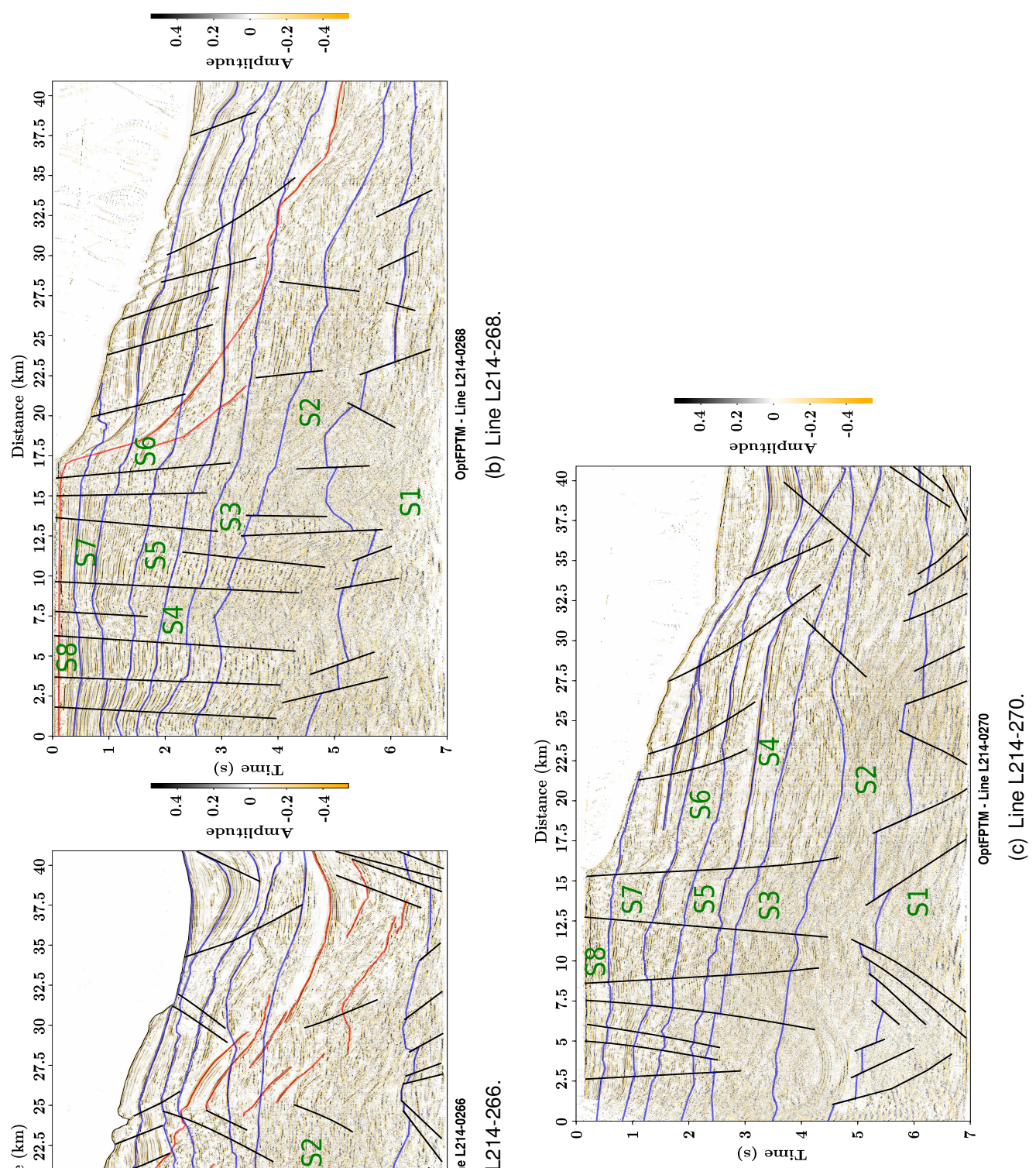

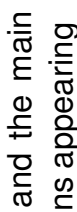

ठิํㅠ

$\cong$ ○

돈

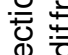

잉

응 호

을 닝

है ญ

. ฮิ ฏ

ब文

$\stackrel{\Phi}{\mp}$

守兵

증

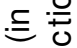

空

Фׁ

兄

$\underline{5}$

ᄃ을

元

음 듣

음

过

के

क के

岁

$\mathrm{z} \infty$

$\stackrel{\oplus}{\risingdotseq}$

$\subseteq \bar{\omega}$

Ð

.

월

$\frac{\omega}{0} \frac{9}{0}$

क्ष

징

흐 으

긍

ఏ.

要

은 을

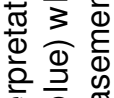

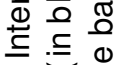

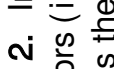

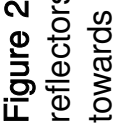



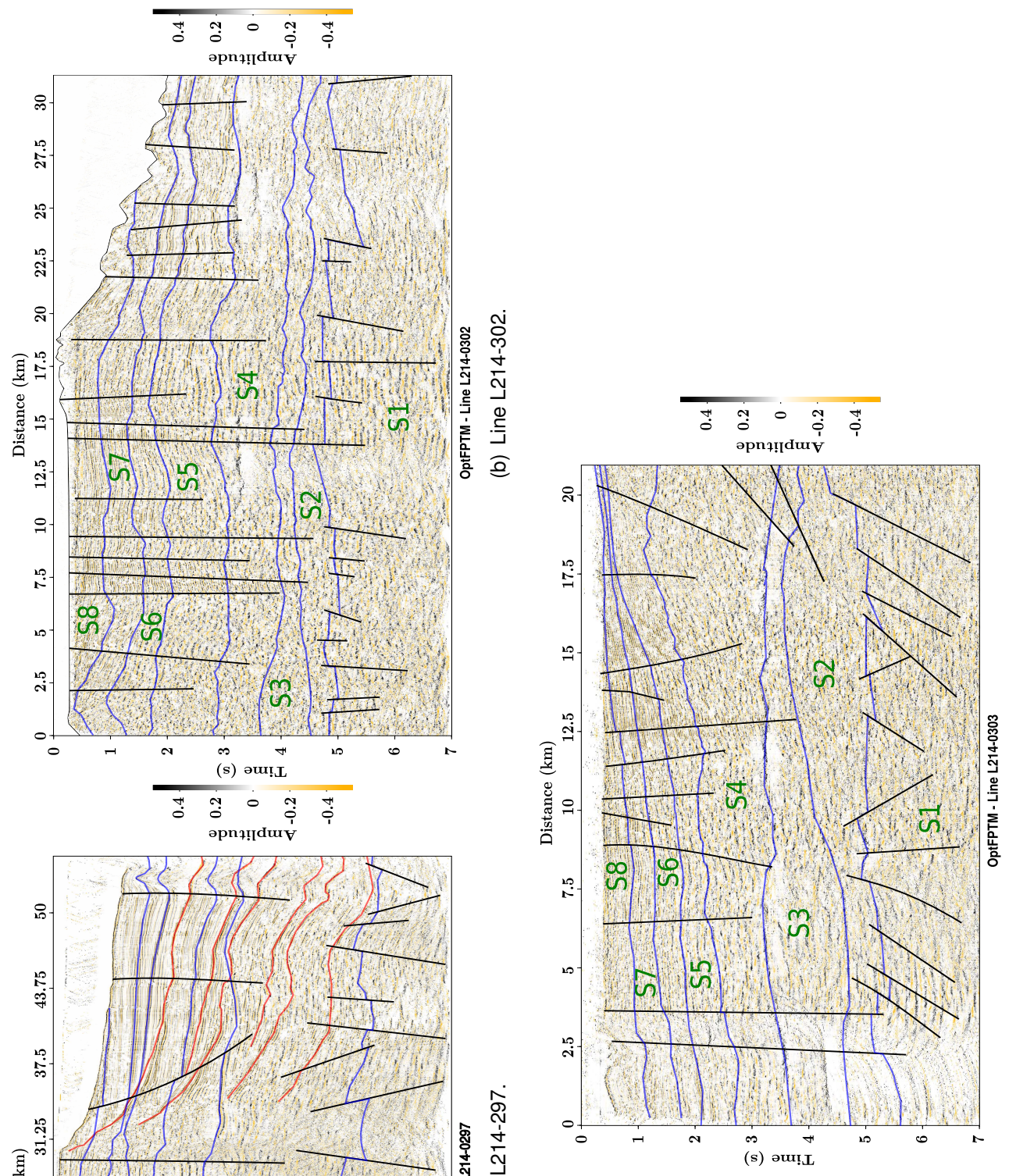

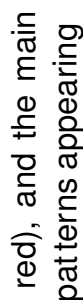

등

든

资

인

응 흔

늘

氜

बे

平

守至

$\frac{\pi}{0} \cdot \frac{0}{6}$

$\subseteq$ 음

क

匹

돓

$\varepsilon .5$

한

을 을

峦

竞

들 일

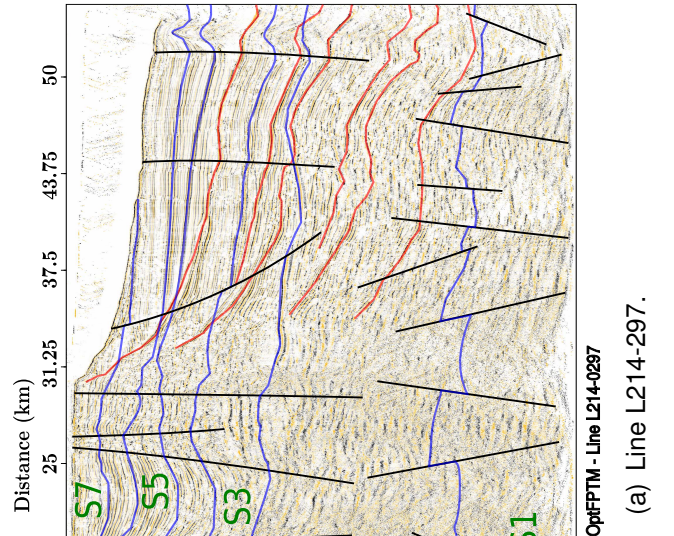

당.

흫

引 के

㟧 $气$

ه

. 우

क人

$\stackrel{9}{=}$

क을

क्ञ

줄 임

흥

을

$\stackrel{\square}{\rightleftarrows}$

잉

든 훙

要

흥

일

的要

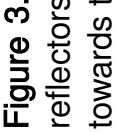


The proper physical description for the geology is according to the mechanics of solids. Then, we consider the rock formations as a set of continuous media, linear elastic, where the stress field, $\sigma_{i j}=\sigma(x, y, z)$, and deformation field, $\epsilon_{i j}=\epsilon(x, y, z)$, are related by Hooke's law as tensors functions of space $(x, y, z)$, which are represented by nine components. Besides, for the general anisotropic media, the tensors $\sigma_{i j}$ and $\epsilon_{i j}$ obey the spatial coordinate rotation by the relations $\sigma_{i j}=\sum_{k, l} a_{i j k l} \sigma_{k l}^{\prime}$, and $\epsilon_{i j}=\sum_{k, l} b_{i j k l} \epsilon_{k l}^{\prime}$, where the coefficients $a_{i j k l}$ and $b_{i j k l}$ define a new plane with respect to a reference system (see Fig. 4).

The elastic linear relationship between stress and strain is given by the generalized Hooke's law: $\sigma_{i j}=$ $\sum_{k, l} c_{i j k l} \epsilon_{k l}$. In this description, the first index $(i)$ in $\sigma_{i j}$ and $\epsilon_{i j}$ represents the plane direction, and the second $(j)$ the component direction.

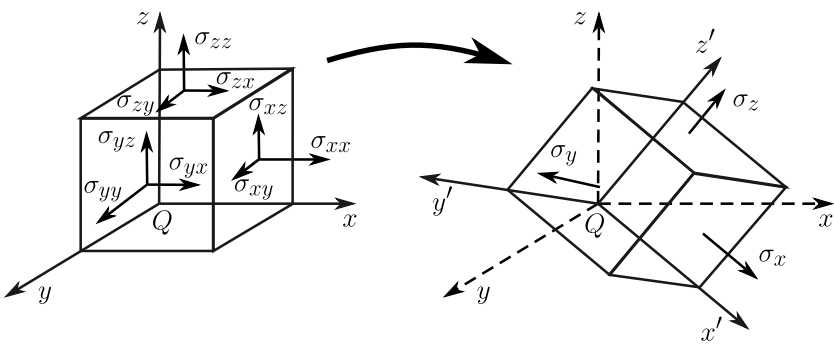

Figure 4. (Left) Illustration of the general stress tensor relative to an infinitesimal c ube representing the point $Q$. (Right) Rotation to any angle to satisfy the (geological) structure for the normal stresses Davis \& Selvadurai (1996).

Stress state is represented at a point $Q$ by a matrix $\mathbf{S}$ with elements $\sigma_{i j}$ (see Fig. 4), that can be decomposed into three parts, $\mathbf{S}=\mathbf{S}_{\mathbf{0}}+\mathbf{S}_{\mathbf{D}}+\mathbf{S}_{\mathbf{N}}$, which allow a physical interpretation, as described by Persen (1975). For the state $\mathbf{S}_{\mathbf{0}}$, we have $\mathbf{S}_{\mathbf{0}}=\left\{P_{R} \delta_{i j}\right\}$, where the diagonal $P_{R}=\frac{1}{3}\left(\sigma_{x x}+\sigma_{y y}+\sigma_{z z}\right)$ represents the simple average of the normal stresses (average or the first stress invariant) which is used to define the so-called rock (solid) pressure (similar concept to the hydrostatic state and, therefore, taken as scalars).

For the state $\mathbf{S}_{\mathrm{D}}$ we have that:

$$
\mathbf{S}_{\mathbf{D}}=\left[\begin{array}{ccc}
\sigma_{x x}-P_{R} & \frac{1}{2}\left(\sigma_{x y}+\sigma_{y x}\right) & \frac{1}{2}\left(\sigma_{x z}+\sigma_{z x}\right) \\
\frac{1}{2}\left(\sigma_{x y}+\sigma_{y x}\right) & \sigma_{y y}-P_{R} & \frac{1}{2}\left(\sigma_{y z}+\sigma_{z y}\right) \\
\frac{1}{2}\left(\sigma_{x z}+\sigma_{z x}\right) & \frac{1}{2}\left(\sigma_{z y}+\sigma_{y z}\right) & \sigma_{z z}-P_{R}
\end{array}\right] .
$$

Applying the symmetry property to the above matrix (1): $\sigma_{x y}=\sigma_{y x}, \sigma_{x z}=\sigma_{z x}, \sigma_{y z}=\sigma_{z y}, \mathbf{S}_{\mathbf{D}}$, results in a state represented by:

$$
\mathbf{S}_{\mathbf{D}}=\left[\begin{array}{ccc}
\sigma_{x x}-P_{R} & \sigma_{x y} & \sigma_{x z} \\
\sigma_{x y} & \sigma_{y y}-P_{R} & \sigma_{y z} \\
\sigma_{x z} & \sigma_{y z} & \sigma_{z z}-P_{R}
\end{array}\right],
$$

what places the deviatoric state along the diagonal (normal stresses), where the "hydrostatic" state is sub- tracted, remaining the non-hydrostatic state. Matrix $\mathbf{S}_{\mathbf{D}}$ is generally called deviatoric state because it represents the degree to which a certain state of tension deviates from the "hydrostatic" state.

For the state $\mathbf{S}_{\mathbf{N}}$, we have that:

$$
\mathbf{S}_{\mathbf{N}}=\left[\begin{array}{ccc}
0 & \frac{1}{2}\left(\sigma_{x y}-\sigma_{y x}\right) & \frac{1}{2}\left(\sigma_{x z}-\sigma_{z x}\right) \\
\frac{1}{2}\left(\sigma_{x y}-\sigma_{y x}\right) & 0 & \frac{1}{2}\left(\sigma_{y z}-\sigma_{z y}\right) \\
\frac{1}{2}\left(\sigma_{x z}-\sigma_{z x}\right) & \frac{1}{2}\left(\sigma_{y z}-\sigma_{z y}\right) & 0
\end{array}\right] .
$$

Similarly, by applying the symmetry property, the state $\mathbf{S}_{\mathbf{N}}$ is simplified to the null state, that is $\mathbf{S}_{\mathbf{N}}=[\mathbf{0}]$.

For an isotropic linear elastic medium, the relation between stress and strain is represented by Hooke's law

$$
\sigma_{i j}=\lambda \theta \delta_{i j}+2 \mu \epsilon_{i j},
$$

where $\lambda$ and $\mu$ are the Lamé elastic parameters, and $\delta_{i j}$ the Kronecker delta $\left(\delta_{i j}=0\right.$, if $i \neq j$ and $\delta_{i j}=1$, if $i=j$ ). The non-dimensional parameter $\theta$ represents the cubic dilation and is given by the divergence of the displacement vector $\mathbf{u}$ as $\theta=\nabla \cdot \mathbf{u}=\frac{\partial u_{x}}{\partial x}+\frac{\partial u_{y}}{\partial y}+\frac{\partial u_{z}}{\partial z}$.

Non-dimensional tensor components $\epsilon_{i j}$ are defined in terms of the displacement components $u_{i}$ in the following form: $\epsilon_{i j}=\frac{1}{2}\left(\frac{\partial u_{i}}{\partial x_{j}}+\frac{\partial u_{j}}{\partial x_{i}}\right)$. Besides that, the linear shear-extensional tensor, a non-dimensional rotation tensor, is also expressed as: $\varphi_{i j}=\frac{1}{2}\left(\frac{\partial u_{i}}{\partial x_{j}}-\frac{\partial u_{j}}{\partial x_{i}}\right)$. We may observe that, once the displacement field is known, the stress and deformation tensors can be calculated by applying spatial derivatives.

\section{Governing Equations}

The system of differential equations that represents static stress-state on a physical particle of the subsurface relates the involved forces in the following way:

$$
\begin{gathered}
\frac{\partial \sigma_{x x}}{\partial x}+\frac{\partial \sigma_{x y}}{\partial y}+\frac{\partial \sigma_{x z}}{\partial z}=p_{x}(x, y, z), \\
\frac{\partial \sigma_{y x}}{\partial x}+\frac{\partial \sigma_{y y}}{\partial y}+\frac{\partial \sigma_{y z}}{\partial z}=p_{y}(x, y, z), \\
\frac{\partial \sigma_{z x}}{\partial x}+\frac{\partial \sigma_{z y}}{\partial y}+\frac{\partial \sigma_{z z}}{\partial z}=p_{z}(x, y, z),
\end{gathered}
$$

where $p_{j}(x, y, z)$ for $j=x$ and $j=y$ may represent the horizontal tectonic (external) stress-pressure fields applied on the block forming the basin. This system must be integrated to obtain the displacement field, from where the stress field can be calculated. For this, it is necessary to define a model and boundary conditions. For $j=z$, we have the vertical gravity loading, $p_{z}=\rho(z) g(z)$, that can be further simplified to $p_{z}=\rho g$ constant for considered thickness column due to small variation of the product $\rho g$ with depth, $z$. For the present approach, the vertical gravity load is considered the only component responsible for the basin stress field. Therefore, the horizontal tectonic components are null, i.e., $p_{x}(x, y, z)=0$ and $p_{y}(x, y, z)=0$.

The present model is related to the wave propagation 
in a perfect linear elastic medium, where the elastodynamic equations of motion have on the right-hand side the term $\rho \frac{\partial^{2} u_{j}}{\partial t^{2}}$ in Eq. (5). To recall, the underlying phenomenon of elastic wave propagation is described by the system of equations (dynamic state) resumed to the form:

$$
\frac{\partial \sigma_{i j}}{\partial x_{j}}=\rho \frac{\partial^{2} u_{i}}{\partial t^{2}} .
$$

This means that the temporal-spatial stress variation is related to the inertial forces (per unit volume), not considering internal forces in this case. It should be clear that we are not studying wave propagation in pre-stressed media, but the case of static deformation, which is analyzed based on the dynamic equation of motion for the limit of frequency zero.

Basic seismic velocities ( $\mathrm{P}$ and $\mathrm{S}$ ) for elastic, homogeneous, isotropic media are given by $V_{P}=\sqrt{\frac{\lambda+2 \mu}{\rho}}$ and $V_{S}=\sqrt{\frac{\mu}{\rho}}$, where $\mu$ is the shear modulus, $\lambda$ is related to the bulk and shear modulus, and $\rho$ is the volumetric density. From the above relations, the modules can calculated by $\mu=V_{S}^{2} \rho, \lambda=V_{P}^{2} \rho-2 \mu=$ $\left(V_{P}^{2}-2 V_{S}^{2}\right) \rho$ and the gamma ratio $\gamma=\frac{V_{S}}{V_{P}}=\sqrt{\frac{\mu}{\lambda+2 \mu}}$. Waves sample the elastic parameters in the subsurface during propagation, and the parameters are considered to vary only spatially, and not with the propagation time.

Density is usually admitted as a parameter that varies slowly with depth, from the earth's surface to the top of the target interface; but, in some situations of complex geology (for instance, presence of magmatic and salt intrusions), the density discontinuity can be relatively high. In the present case of vertical sections, the density is modeled in 2D, $\rho=\rho(x, z)$, and is integrated into the calculation grid.

The system of differential equations to be integrated corresponds to the description of the static problem [no time variation, Eq. (5)]. These equations govern the mechanics of solids under the influence of gravity only, and they are summarized in the form $\frac{\partial \sigma_{3 j}}{\partial x_{j}}=\rho g \delta_{3 j}$. This means that the sum of the horizontal stress variations is zero, and the vertical component is controlled by the gravitational load in the subsurface expressed in terms of force per unit area and set to $p_{z}=\rho g$. Therefore, the lateral tectonic stresses are not considered in this model, besides we do not have such easy information for the area. Also, for other specific cases the quantities $\rho$ and $g$ may be considered as spatial functions, i.e., $\rho=\rho(x, y, z)$ and $g=g(x, y, z)$.

\section{Parameter Integration}

We consider the geological medium as represented by a model formed by plane-horizontal layers along the sampling grid in the $x$-axis, and heterogeneous along the vertical $z$-axis for each point of the profile. Equilibrium equation for the linear elastic medium for each layer is $\frac{\partial \sigma_{i k}}{\partial x_{k}}=\rho g_{i}$, that is simplified to the form $\frac{\partial \sigma_{z z}}{\partial x_{z}}=$ $\rho g$. This equation has an elementary solution given by:

$$
\sigma_{z z}(z)=g \int_{z=0}^{z=z} \rho(z) d z,
$$

that measures the overload rock weight per unit area; i.e., the vertical pressure due to the overload at any depth $z$. It is clear that this summation is straightforward as a discrete form, and considers a flat model in the discrete space grid. Also, for our present data, we have a sampling $\Delta x$ along the seismic line different from the sampling in the transverse direction $\Delta y$.

For the model presentation, we simplify the nomenclature. Vertical stress, $\sigma_{z z}(z)$ is defined as due to the overloading layer; i. e., $\sigma_{z z}=P_{Z}(z)$. On the other hand, the horizontal stress $\sigma_{x x}(z)$, considering that $\sigma_{y y}=\sigma_{x x}$, is smaller than the vertical stress, $\sigma_{z z}$, and we can demonstrate to be given by:

$$
\sigma_{x x}(z)=P_{X}(z)=P_{Z}(z)\left[1-2 \gamma^{2}(z)\right],
$$

where $\gamma(z)=\frac{V_{\mathrm{S}}(z)}{V_{\mathrm{P}}(z)}$.

Scalar invariant field $P_{R}(z)$ was defined in the matrix (1) as the average of the first invariant $\left[I_{1}=\sigma_{x x}+\sigma_{y y}+\right.$ $\left.\sigma_{z z}\right]$, namely:

$$
P_{R}=\frac{1}{3}\left(\sigma_{x x}+\sigma_{y y}+\sigma_{z z}\right) .
$$

Using Hooke's generalized law, we can demonstrate that $P_{R}=\left(\lambda+\frac{2}{3} \mu\right) \theta$, where $\theta(z)$ is the cubic dilation, and $\lambda(z)$ and $\mu(z)$ are Lamé's parameters described above. Condition of pure hydrostatic state $P_{H}$ is physically defined for fluids only; in the case of $\sigma_{x x}=\sigma_{y y}=$ $\sigma_{z z}$, then mathematically $P_{R}=P_{H}$, but that is not the usual case for solids under the gravity loading.

Another important physical feature is the pressure discontinuity at the interfaces between different layers ( $\Delta P=P^{+}-P^{-}$, lower minus upper), at the depth $z$ (positive down), which exists if the velocity ratio $\gamma$ exhibits a discontinuity. Considering the case of a medium formed by plane-horizontal layers, and Hooke's isotropic law, we show that the pressure discontinuity $\Delta P$ is given by:

$$
\Delta P(z)=\frac{4}{3}\left(\gamma_{1}^{2}-\gamma_{2}^{2}\right) P_{Z}(z)
$$

where $\gamma_{1}$ is the upper layer and $\gamma_{2}$ the lower layer parameter, across the interface positioned at the depth $z$, which shows that the overload pressure can vary by positive and negative jumps Sibiryakov et al. (2019). This idea may seem a little strange for geological descriptions, but it is a fact related to the non-elementary behavior of stress in solids. As a partial conclusion, interfaces should be the goals in the geological interpretation of the seismic sections, whose examples are shown in Figures 2 and 3.

The intensity of the so-called tangential stress $\left(P_{T}\right)$ is a way of measuring the mechanical instability responsible for the condition of destroying the granular structure, 
to produce fracture in the solid rock. In this direction, the following relation is obtained:

$$
P_{T}(z)=\frac{1}{2}\left(\sigma_{z z}-\sigma_{x x}\right)=\gamma^{2}(z) P_{Z}(z) .
$$

Once more, this result also depends on the $\gamma$ ratio.

\section{Modeling Strategy}

The modeling strategy for choosing the physical parameters of the reservoir has to consider a sequence of geological formations forming the stratigraphical units interpreted in the seismic sections. For this purpose, we follow the work of Gregory (1976) and described in detail by Sibiryakov et al. (2019).

Gregory (1976)'s experiments allowed dividing rock porosity into classes: (1st) the class where the velocity behavior does not contradict qualitatively with FrenkelBiot theory, i.e., shear wave velocities decrease with the increase of water saturation; (2nd) the class where there is a qualitative contradiction with Frenkel-Biot theory, i.e., shear wave velocities increase with the increase of water saturation; (3rd) the class where the velocity is pressure-dependent.

An important measure in the mechanics of solids related to pressure prediction and $\gamma$ ratio is Poisson's ratio $\nu$. Among several definitions present in the literature, Poisson's ratio is simply the negative of the transverse strain rate relative to axial deformation in an elastic material subjected to axial stress, that is, $\nu=-\frac{d \epsilon_{y}}{d \epsilon_{x}}$. In terms of Lamé's parameters, Poisson's ratio can be written as $\nu=\frac{\lambda}{2(\lambda+\mu)}$, with a considered usual practical range of values $-1<\nu \leqslant 0.5$ Davis \& Selvadurai (1996). Therefore, the relations between Poisson and $\gamma$ ratios are given by:

$$
\nu=\frac{1}{2} \frac{\left(1-2 \gamma^{2}\right)}{\left(1-\gamma^{2}\right)}, \quad \gamma=\sqrt{\frac{1-2 \nu}{2(1-\nu)}},
$$

plotted in Figure 5, where we observe the points of discontinuity and the general behavior of these two relations, that are inverse of each other. These figures intend to show the general mathematical behavior of relations in Eqs. (12), and not laboratory or field experiments. In other words, we open the possibilities that field and laboratory measurements be different, and to serve as physical bounds. In Figure 5 we highlight the infinite discontinuity of the Poisson's ratio, $\nu(\gamma)$, around the value $\gamma^{2}=1$ (it is not admitted $\gamma<0$ ). On the other hand, the gamma ratio, $\gamma(\nu)$, has a complex discontinuity around the value $\nu=\frac{1}{2}$, and an infinite discontinuity around $\nu=1$; the range for $\frac{1}{2}<\nu<1$ gives the $\gamma$ ratio a complex value.

The most important Gregory (1976)'s result is the experimental detection of abnormal high $\gamma=\frac{V_{S}}{V_{P}}$ ratios for high porosity rocks. If this $\gamma$ ratio exceeds $\frac{1}{\sqrt{2}} \approx$ 0.707 , then, we have $\lambda<0$, and as a result, Poisson's ratio has negative value (see Fig. 5). It has long been considered that Poisson's ratio can only have positive values in the range between 0 and $\frac{1}{2}$. Negative values of Poisson's ratio, in particular, mean that stretching a thin rod by its end faces does not decrease in thickness, but on the contrary, its thickness increases.

Besides, Gregory (1976)'s result is not strange at all, because it does not contradict the fundamentals of thermodynamics about the deformation of a body that demands positive free energy $F$; i.e., positive values calculated according to the formula $F=\mu\left(\epsilon_{i k}-\frac{1}{3} \delta_{i k} \epsilon_{l l}\right)^{2}+$ $\frac{K}{2} \epsilon_{l l}^{2}$. This means that positive values should be for $\mu$ and $K=\lambda+\frac{2}{3} \mu$, but not for $\lambda$; hence, Poisson's ratio is not strictly given by positive values Sibiryakov et al. (2019). Also, we are dealing with a dynamic measure (wave propagation) and not a static measure (laboratory measurements).

Gregory (1976) found in dynamic measurements in laboratory, and other authors in field experiments, abnormally high relationships of $\gamma=\frac{V_{S}}{V_{P}}$ up to 0.77 . Static measurements give positive values for the Poisson and gamma ratios. But, there is no complete clearness concerning reasons for such a phenomenon as described by Sibiryakov et al. (2019). For this reason, we identified the reservoir formation with the value $\gamma=0.807$ chosen arbitrarily.

\section{RESULTS}

\section{Input and Calculated Parameters}

Distributions $V_{P}$ and $\gamma$ ratio, used as input parameters, are based on controlled empirical models with values presented in Table 1. In a real and practical case, where a good velocity analysis can be performed, empirical models are less important; as a matter of fact, velocity and density estimations are the main challenges in seismic processing.

$V_{P}$ sections displayed in Figure 6 have the main layers indicated by letters and numbers in the following order: S9 represents the water layer (dark blue), S4 the layer above the reservoir (light blue), S3 the layer that includes the reservoir (light green), S2 the layer that includes the source rock (yellow), and S1 the basement (dark red).

In each layer, $V_{P}$ was defined by the formula $V_{P}(z)=$ $V_{P 0}+k_{P} z$ with a small linear gradient $k_{P}$. The $\gamma$ ratio is also defined as a linear function, $\gamma(z)=\gamma_{0}+k_{g} z$, with also a small gradient $k_{g}$. For the density $\rho$, we used the classical empirical relation $\rho(z) \approx 1.741 V_{S}^{0.25}(z)$ proposed by Gardner et al. (1974), which represents an average on different types of rock, but that should attend Birch's law. These empirical models were applied to the interpreted seismic-stratigraphic sections of the Figures 2 and 3 , along the $z$-axis and in each point of the $x$-axis, producing 2D maps for distributions $V_{P}(x, z), V_{S}(x, z)$, $\rho(x, z)$ and $\gamma(x, z)$.

The initial information for the pressure prediction is the sections of the velocity $V_{P}$ and $\gamma$ ratio, from where the other parameters are calculated (see Figs. 6, 7, 8, and 9). To obtain these sections of parameter distributions, we started from the CRS migrated and interpreted 

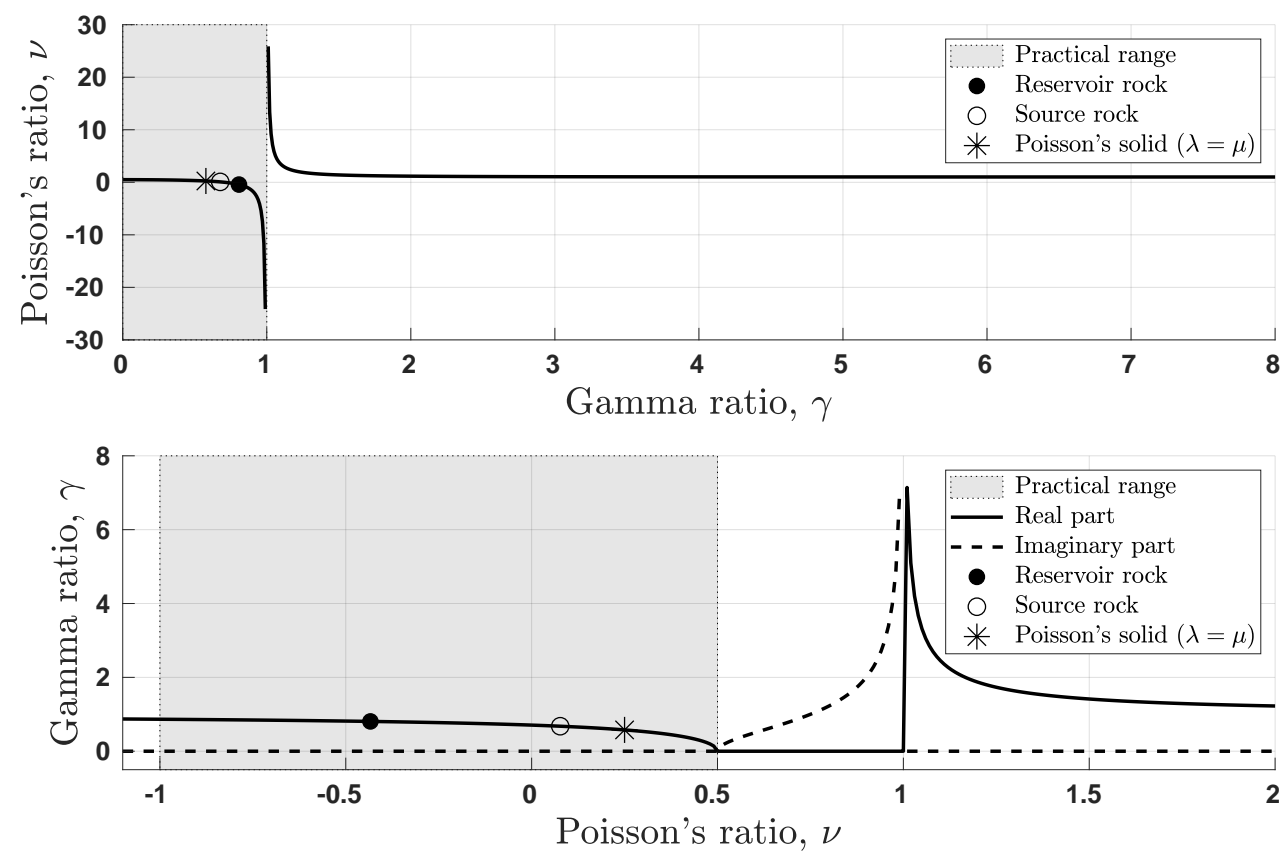

Figure 5. Graphs of the relationships (12) between Poisson and gamma ratios for a range of theoretical values. We marked the reservoir (S3), the source rock (S2), and the Poisson's solid values in both graphs. The light gray and white areas correspond, respectively, to practical and purely theoretical values.

seismic sections, where the source and reservoir formations were defined based on some prior geological information.

It is important to mention that the data was formatted in a matrix form. Therefore, we can apply a simple change of scale from time $(t)$ to depth $(z)$ using a constant average velocity value (in this case $V=1 \mathrm{~m} / \mathrm{s}$ ). In other situations, we can use the data matrix resulting from another process like migration.

The $V_{P}$ empirical models are mixed, i.e., composed of jumps and smooth linear trend variation as displayed in Figure 6, where the gradient varied as $0.01<k_{P}<$ 0.075. A similar formulation was applied to the $\gamma(z)$ distribution, but with an even smoother gradient, with $k_{P}=0.001$ (see Fig. 7). Having defined the $V_{P}(x, z)$ and $\gamma(x, z)$ distributions, $V_{S}(x, z)$ was calculated as shown in Figure 8. As a consequence, the other desired parameter distributions $(\rho, \mu, \lambda, \nu, K)$ can be automatically calculated, followed by the desired pressure sections. Figure 9 shows the density distributions, $\rho(x, z)$, which also followed the mixed pattern similar to $V_{P}$; that is, with jumps and smooth vertical gradients.

\section{Maps and Cubes of the Pressure Predictions}

\section{Maps}

The following figures show the section maps for $P_{Z}$, $P_{X}, P_{T}$, and $P_{R}$, where we focus our attention on the low- and high-pressure zones. Figure 10 shows the pressure $P_{Z}(x, z)$ (vertical load), which presents a very smooth behavior as expected. Figure 11 shows the pressure $P_{X}(x, z)$ (horizontal component), which exhibits a very differentiated and special behavior for each seismic section, where zones of low negative pres- sure contrast can be seen for each layer interpreted as the reservoir (light green range between 2000 and 4000 meters). Figure 12 shows the deviatoric pressure $P_{T}$ between vertical and horizontal stress components, with a very special behavior for the low- and high-pressure zones, and clear information that the vertical pressure exceeds the horizontal pressure in general. Besides that, it is interesting to observe that part of the basement presents zones of intermediate pressure.

Figure 13 shows the rock pressure $P_{R}$, where it is clearly modeled the low-pressure zone (central blue stripe), located between two high-pressure zones (weaker above and stronger below).

\section{Cubes}

Pressure cubes were constructed by linear interpolation of the six seismic lines used with the arrangement already shown in Figure 1. The lines form two groups (NW-SE and NE-SW), where each group is formed by three parallel lines. Initially, the pressure maps (rock, tangential, and horizontal) were bounded to equalize the sizes of the lines. Then, we established an equal spatial sampling along all six lines $(\Delta x=25 \mathrm{~m})$ and built a cube for each group. After that, the cubes were decimated by 20 points and then merged into a single cube. The matrix representing the cube had $84 \times 84 \times 120$ points, and its 3D grid had the spatial sampling $\Delta x=504.8 \mathrm{~m}, \Delta y=144.6 \mathrm{~m}$, and $\Delta z=58.6 \mathrm{~m}$. The cube gives the possibility to rotate it, navigate inside, and do different cuts, which produces a more convenient image for interpretation and to follow the continuation of the reservoir inside the cube. Nevertheless, the structural analysis must consider the limitations of 
Table 1. Parameter values used in the empirical models.

\begin{tabular}{c|c|c|c|c|c|c|c|c|c}
\hline Value & S9 & S8 & S7 & S6 & S5 & S4 & S3 & S2 & S1 \\
\hline$\gamma_{0}$ & 0 & 0.677 & 0.677 & 0.677 & 0.677 & 0.687 & 0.807 & 0.677 & 0.570 \\
$V_{P 0}$ & 1500 & 1900 & 2200 & 2350 & 2500 & 3000 & 3440 & 4200 & 5200 \\
$k_{P}$ & 0 & 0.01 & 0.01 & 0.01 & 0.05 & 0.02 & 0.025 & 0.075 & 0.2 \\
$k_{g}$ & 0 & 0.0001 & 0.0001 & 0.0001 & 0.0001 & 0.0001 & 0.0001 & 0.0001 & 0.0001 \\
\hline
\end{tabular}

asymmetric sampling.

Figure 14 shows an important 3D cube constructed for the rock pressure, $P_{R}(x, y, z)$. This cube highlights the zone interpreted as a reservoir (dark blue in the middle of the cube), which corresponds to the lowest pressure zone. This image helps us to extend threedimensionally the zone containing the possible productive reservoir.

Figure 15 shows the 3D cube constructed for the horizontal pressure $P_{X}(x, y, z)$, where we can notice the spatial distribution of the low-pressure zones across the intermediate part of the cube. Figure 16 shows the $3 \mathrm{D}$ cube constructed for the tangential pressure $P_{T}(x, y, z)$, where the highest deformations that can take place are located along the orange-red zones.

\section{CONCLUSIONS}

Our purpose in this work was sedimentary basin modeling through methods of pressure prediction to map lowand high-pressure zones that act as natural pumps for fluid accumulation, and more specifically for oil and gas exploration. Low-pressure zones mapped in the subsurface should serve as targets for the location of important drilling as an aid to geological decisions.

The seismic data were processed using the CRS technology because it showed to be more effective for the geometrical delineation of interfaces (reflectors). Stacked and migrated sections resulting from the CRS processing were used to build empirical models of velocity and density. Besides, the work is a demonstration that it is possible to map low- and high-pressures zones using the seismic information of velocities $V_{P}$ and $V_{S}$, and density $\rho$.
It is interesting that the imaging process for mapping low-pressure zones, corresponding to a proposed (or productive) reservoir, can have a spatial extension that can be updated with further seismic, stratigraphic, and structural information of the sedimentary basin. This means that pressure prediction is a systematic interactive process in basin modeling so here defined, because a real knowledge of $V_{P}, V_{S}$, and $\rho$ is the greatest want of all seismic research.

Rock "hydrostatic" pressure, defined as an average of the normal stresses, corresponds to a convenient definition based on the first invariant for the stress-strain state of solids. Therefore, although it does not have real physical meaning, it represents a powerful concept and has strong mathematical reasoning.

Furthermore, we showed that the concept of a sealing cap rock does not need to be associated with lithological and structural considerations, but mainly with the condition of low- and high-pressure zones related to the present geological environment. Namely, a reservoir tends to be a low-pressure zone and the cap-rock a high-pressure zone. This fundamental conclusion is a reality of the physics of solids, but that can sound strange to geologists as we found from our experience during oral presentations.

In conclusion, a further continuation of this method requires combined data from $3 \mathrm{D}$ seismic surveys with borehole, geochemical, geological information, to compose a more realistic 3D model for pressure prediction and, therefore, show a possible new reservoir or to extend a productive reservoir. 


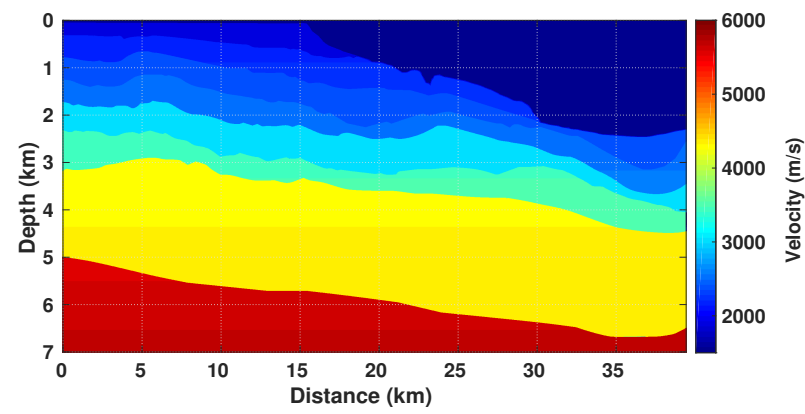

(a) Line L214-266.

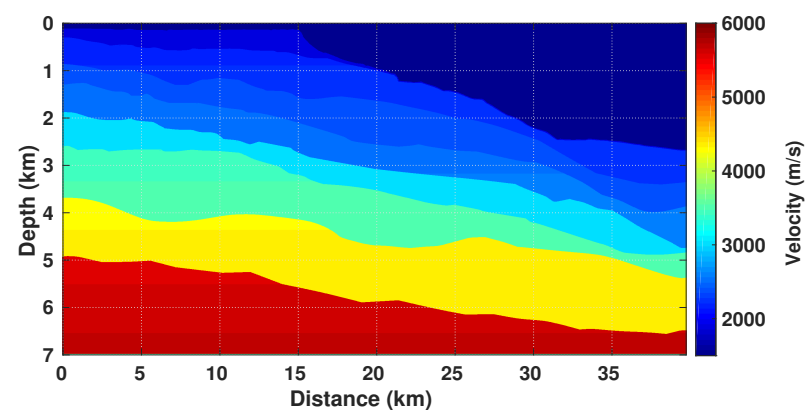

(c) Line L214-270.

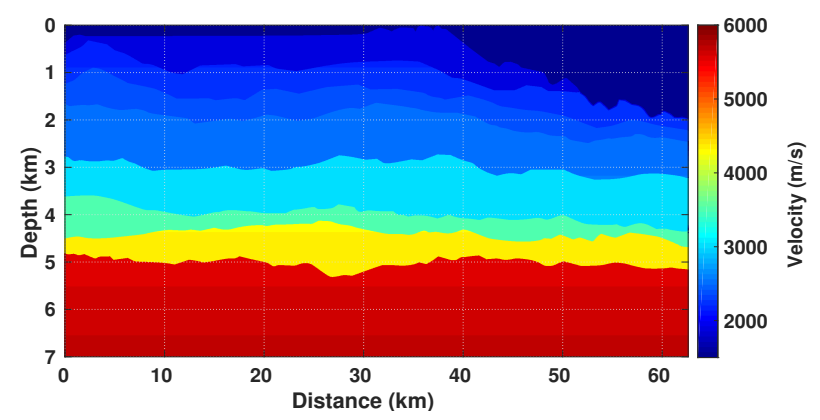

(e) Line L214-302.

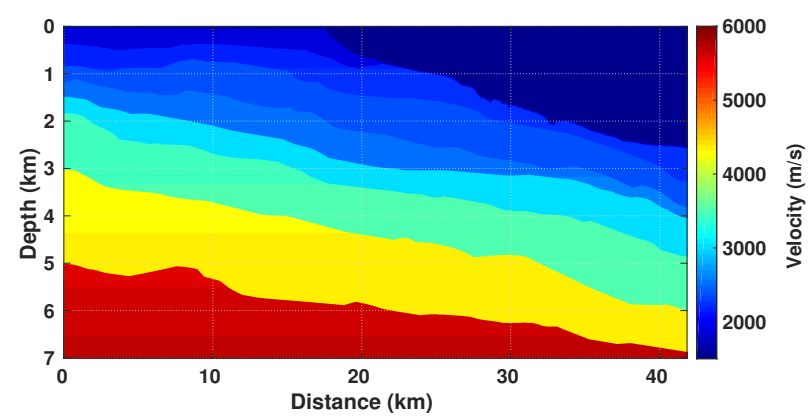

(b) Line L214-268.

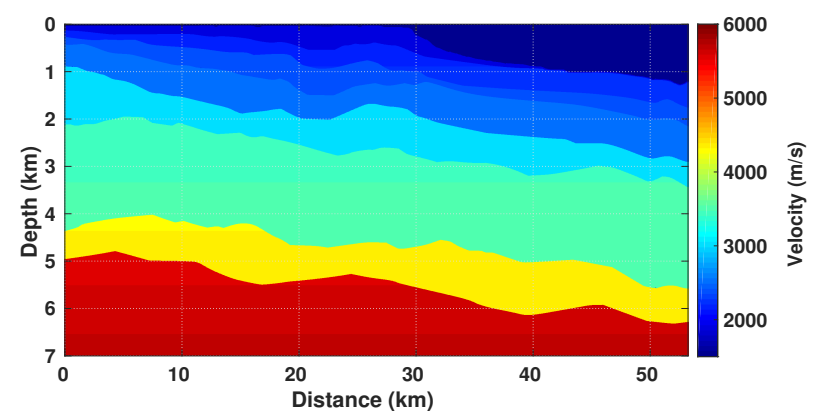

(d) Line L214-297.

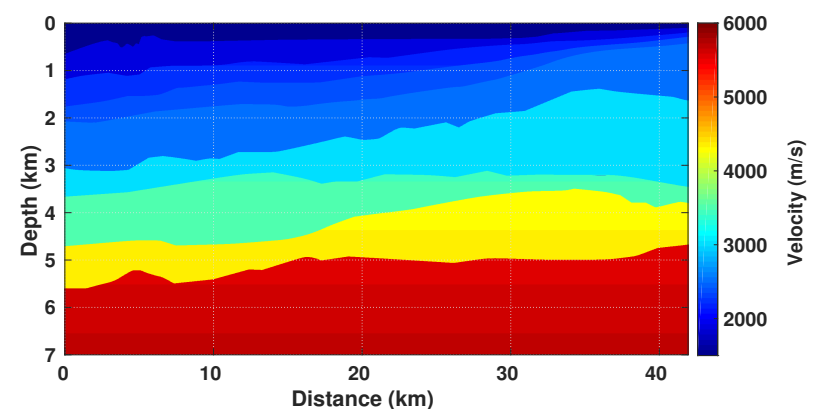

(f) Line L214-303.

Figure 6. P-wave velocity distributions $V_{P}(x, z)$. 


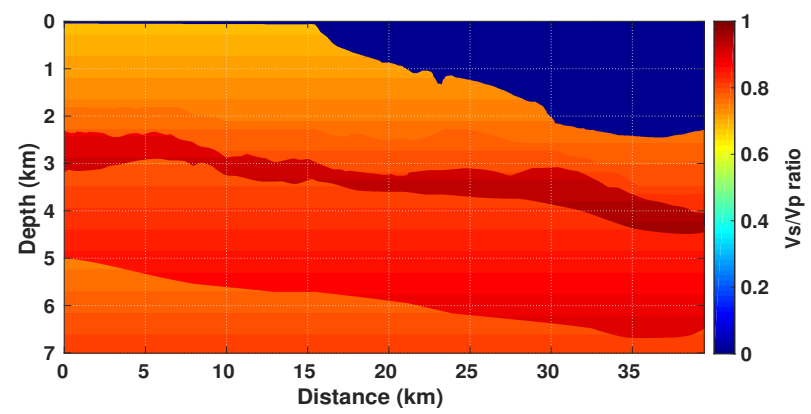

(a) Line L214-266.

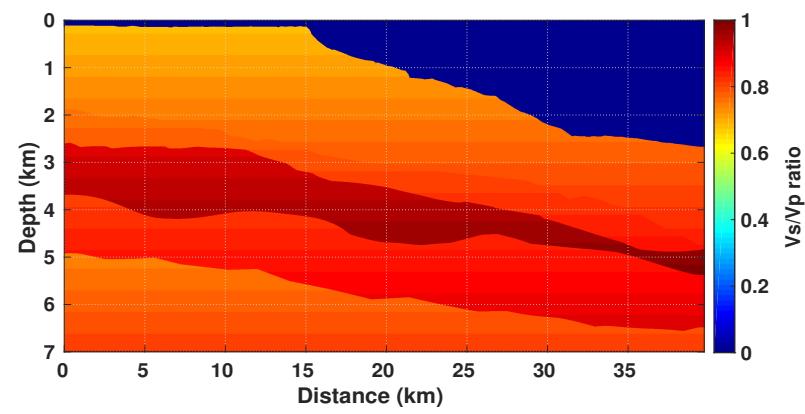

(c) Line L214-270.

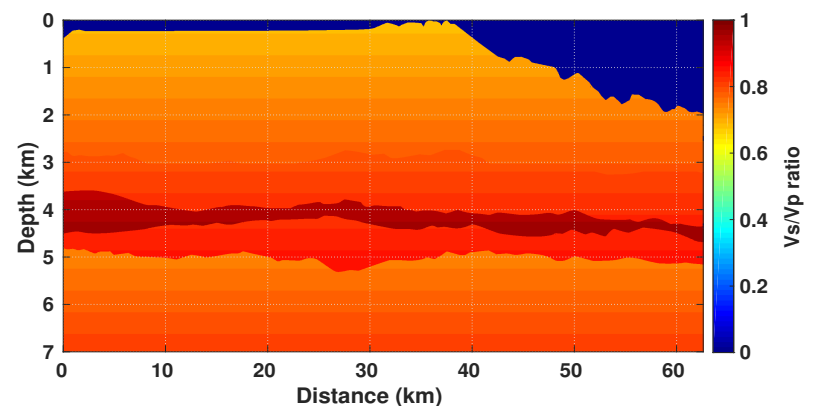

(e) Line L214-302.

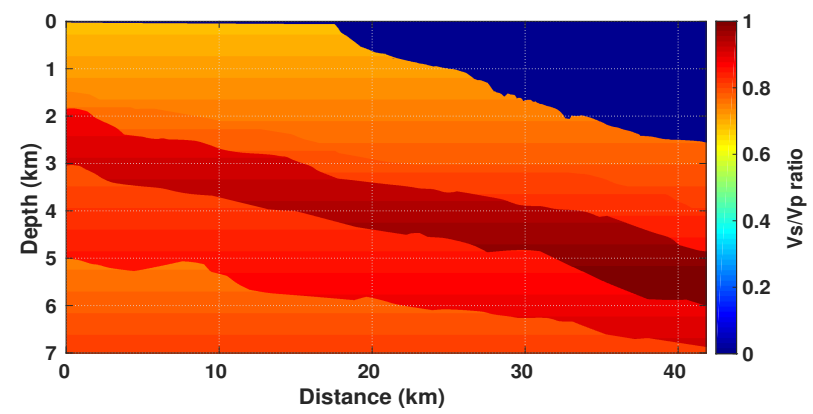

(b) Line L214-268.

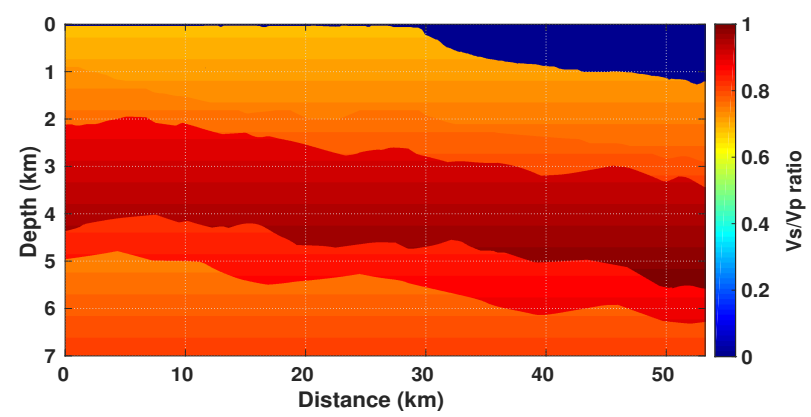

(d) Line L214-297.

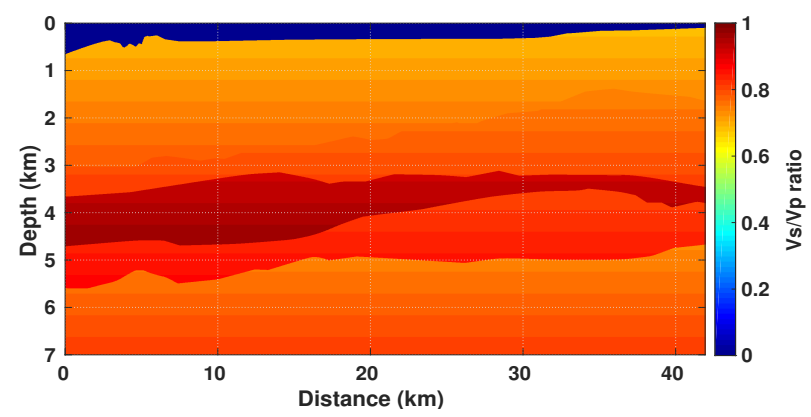

(f) Line L214-303.

Figure 7. Gamma ratio distributions $\gamma(x, z)$. 


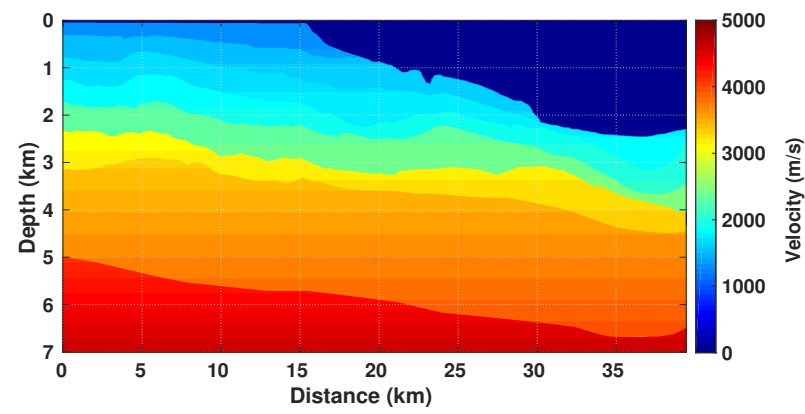

(a) Line L214-266.

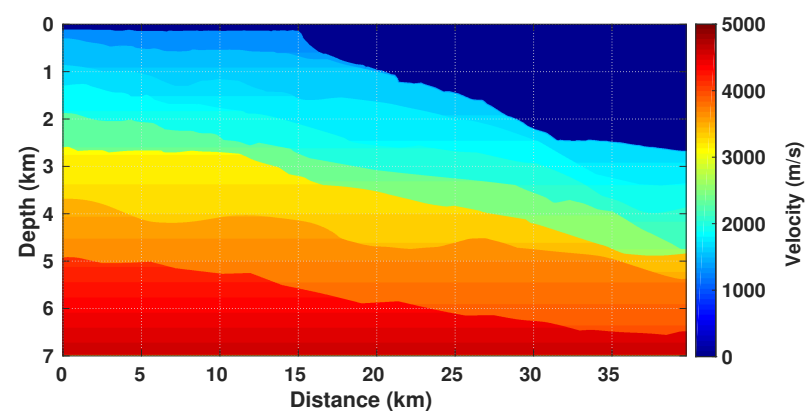

(c) Line L214-270.

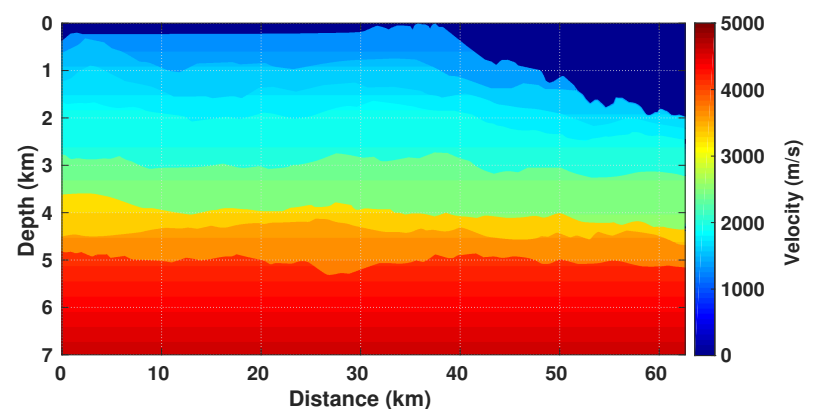

(e) Line L214-302.

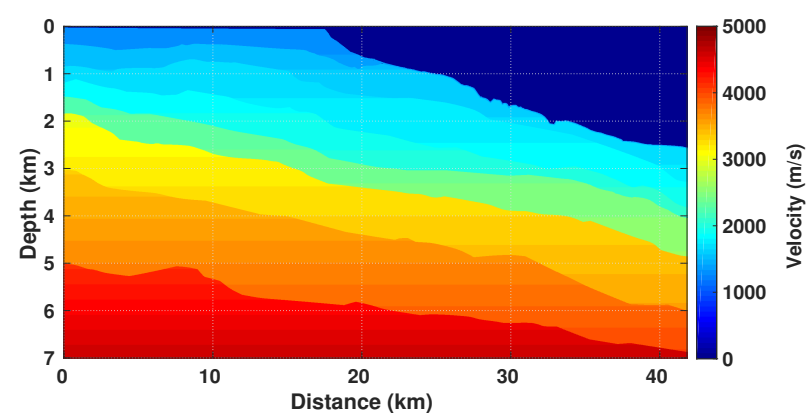

(b) Line L214-268.

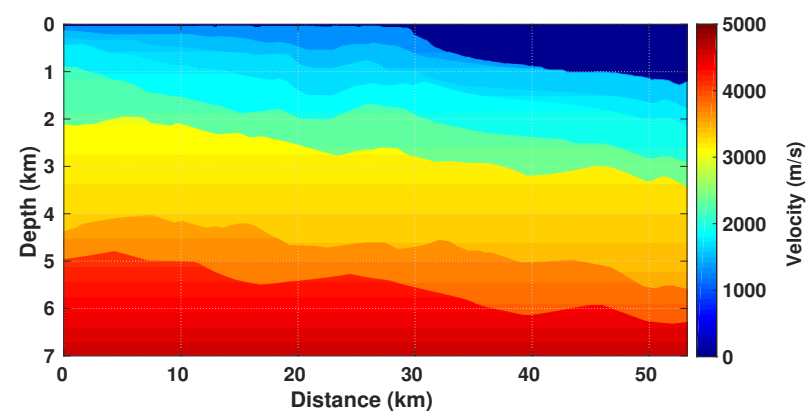

(d) Line L214-297.

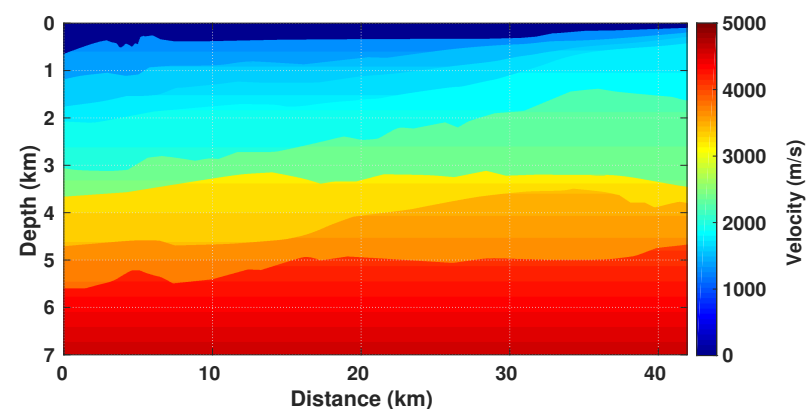

(f) Line L214-303.

Figure 8. S-wave velocity distributions $V_{S}(x, z)$. 


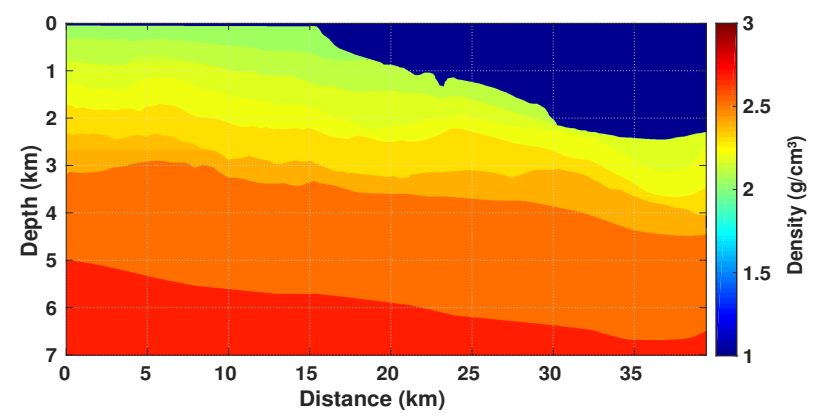

(a) Line L214-266.

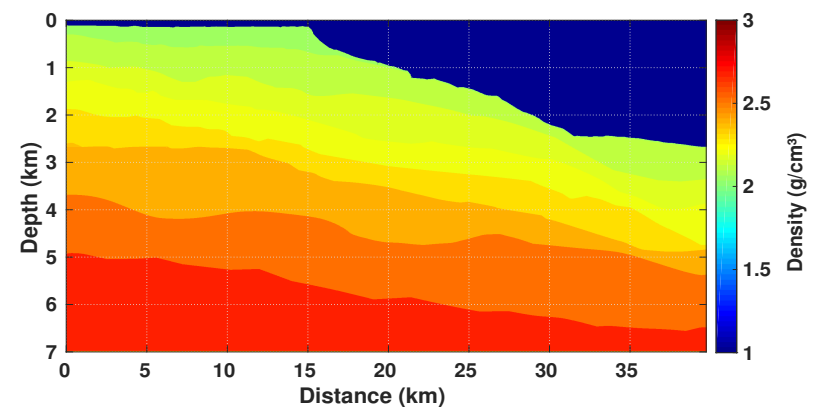

(c) Line L214-270.

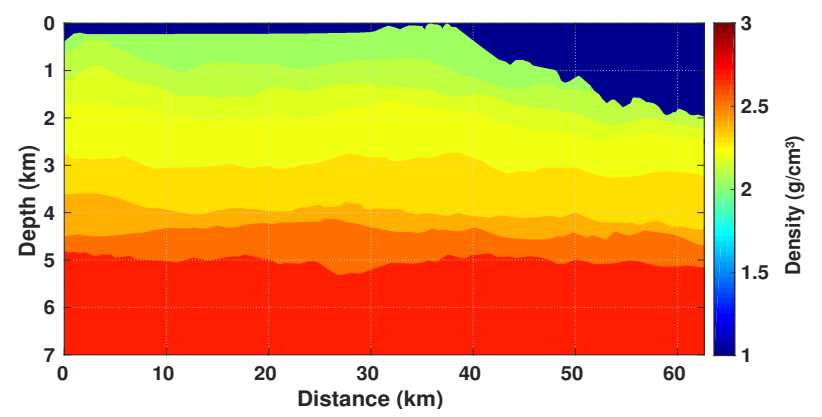

(e) Line L214-302.

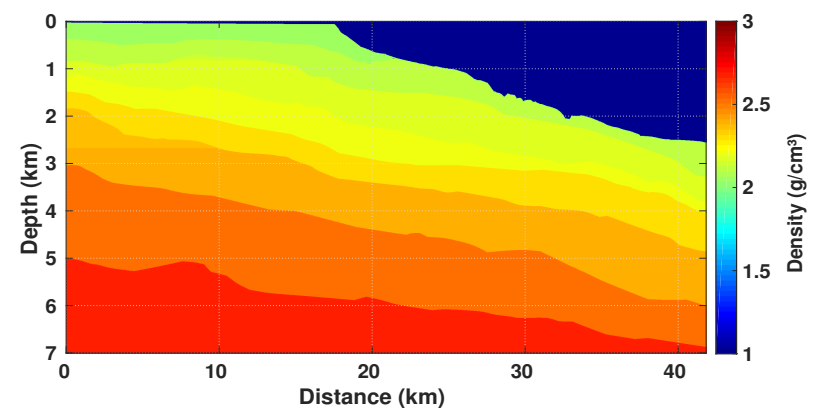

(b) Line L214-268.

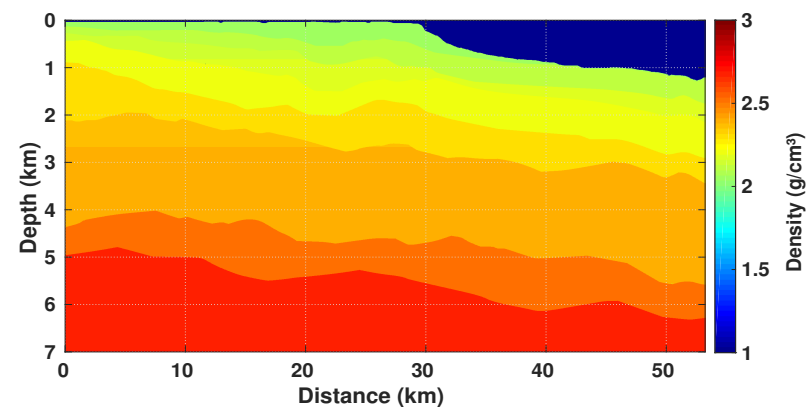

(d) Line L214-297.

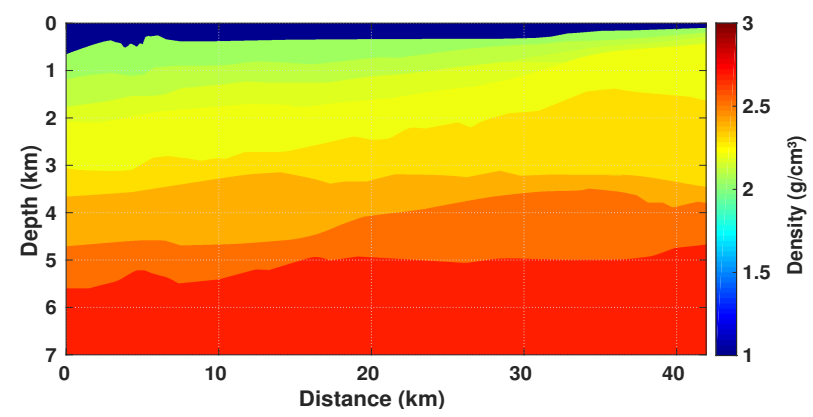

(f) Line L214-303.

Figure 9. Density distributions $\rho(x, z)$. 


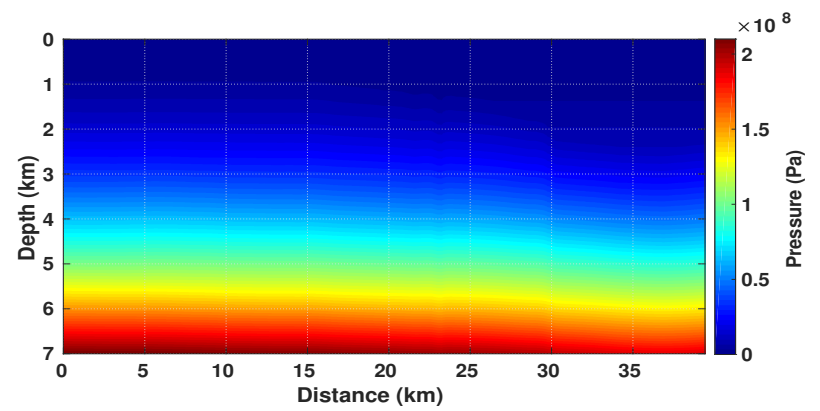

(a) Line L214-266.

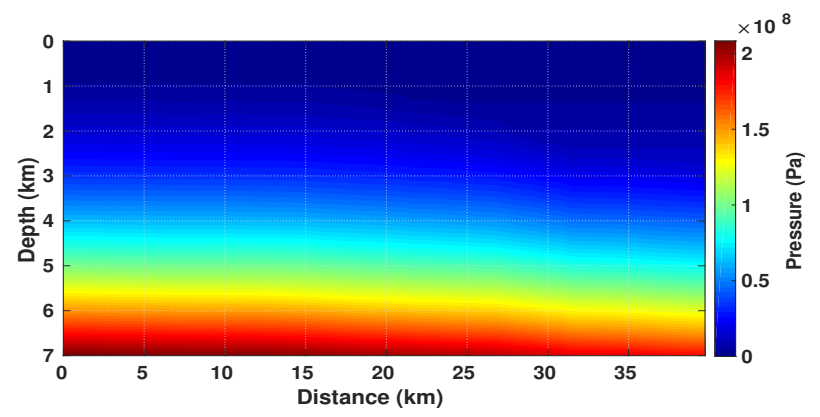

(c) Line L214-270.

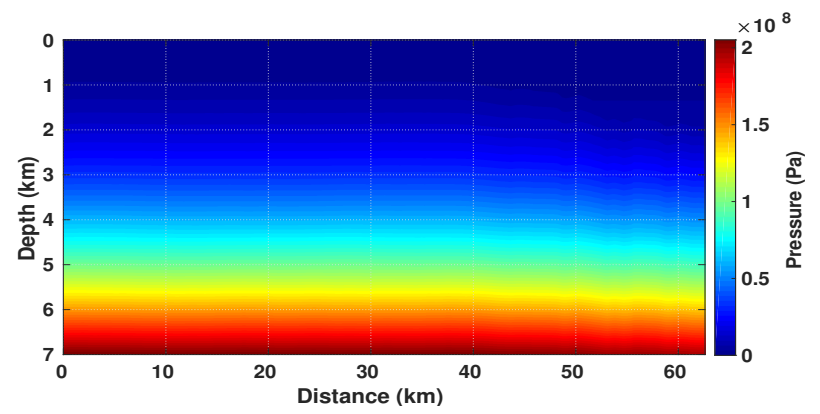

(e) Line L214-302.

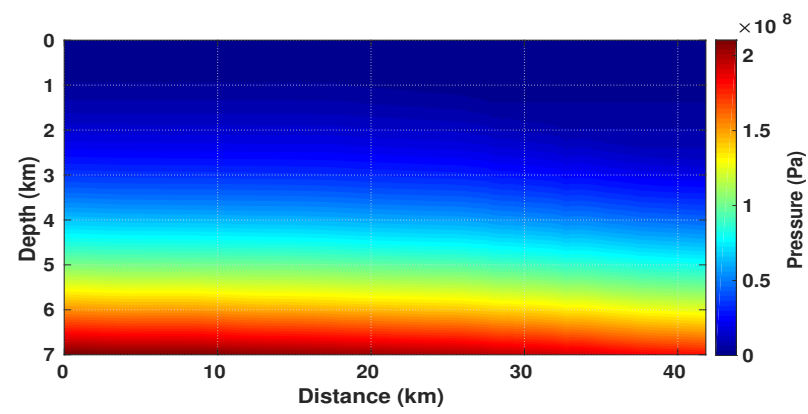

(b) Line L214-268.

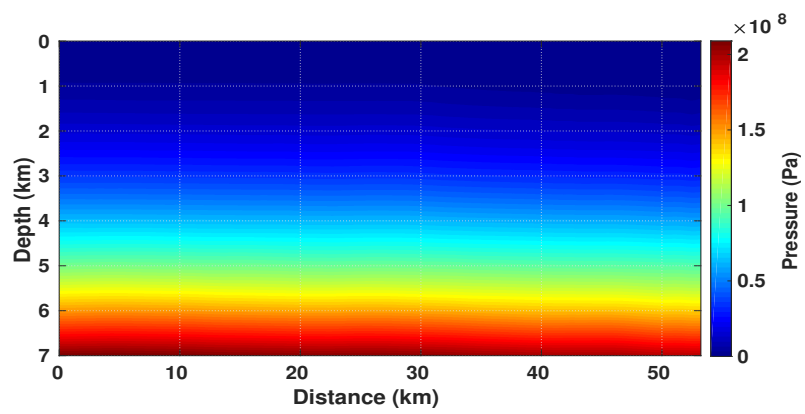

(d) Line L214-297.

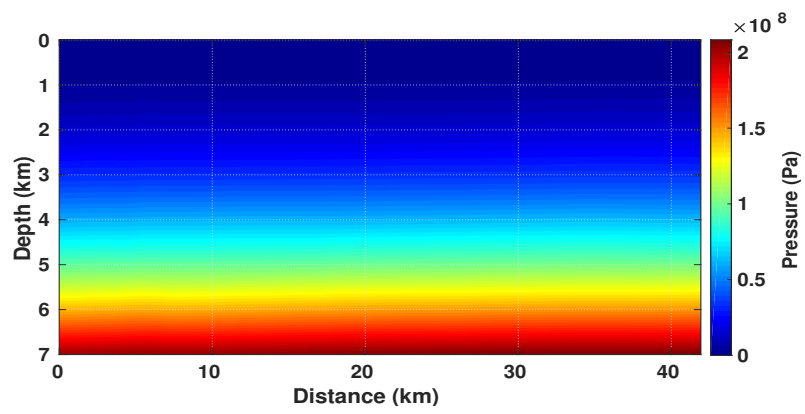

(f) Line L214-303.

Figure 10. Maps of vertical pressure $P_{Z}(x, z)$. 


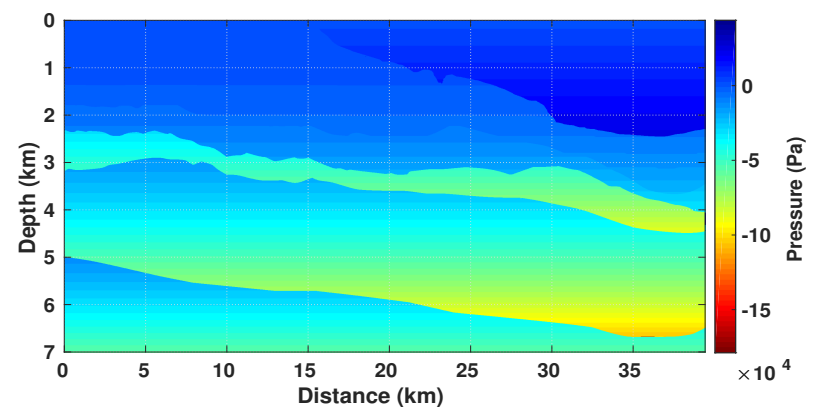

(a) Line L214-266.

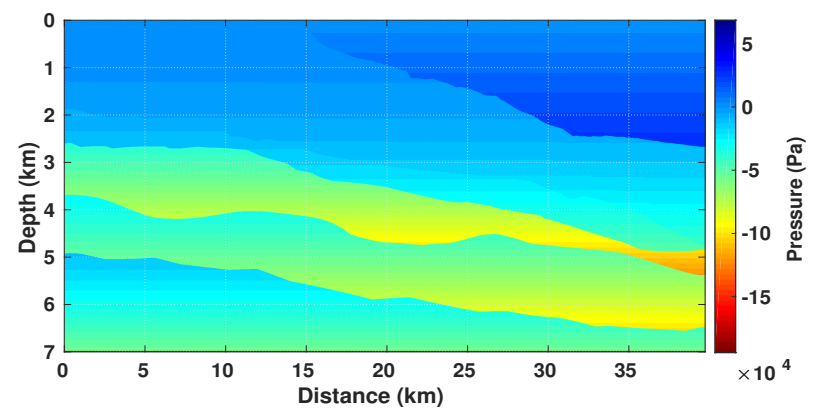

(c) Line L214-270.

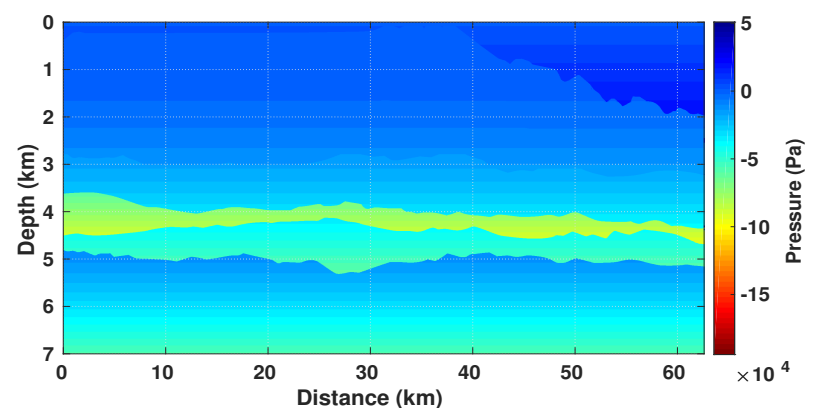

(e) Line L214-302.

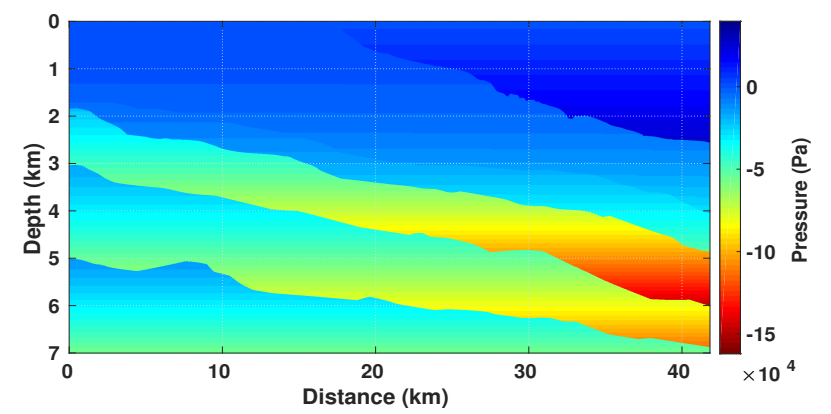

(b) Line L214-268.

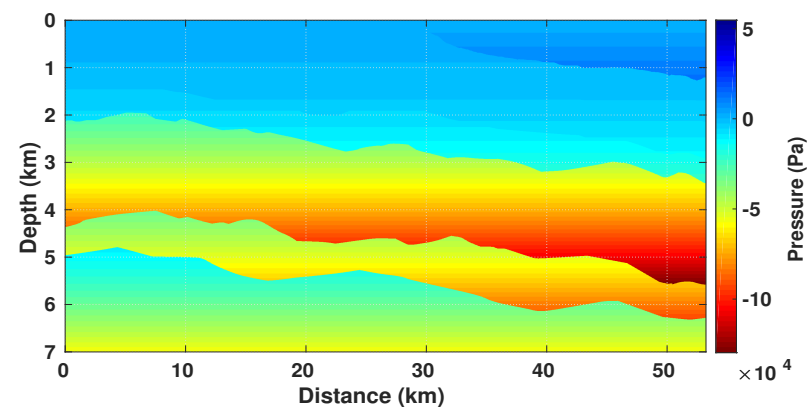

(d) Line L214-297.

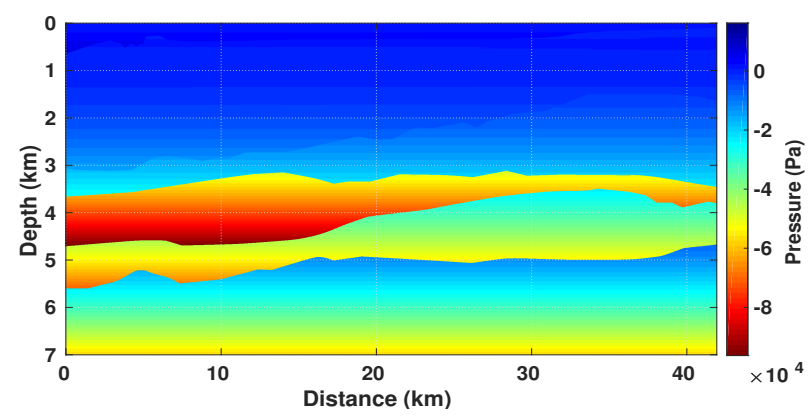

(f) Line L214-303.

Figure 11. Maps of horizontal pressure $P_{X}(x, z)$. 


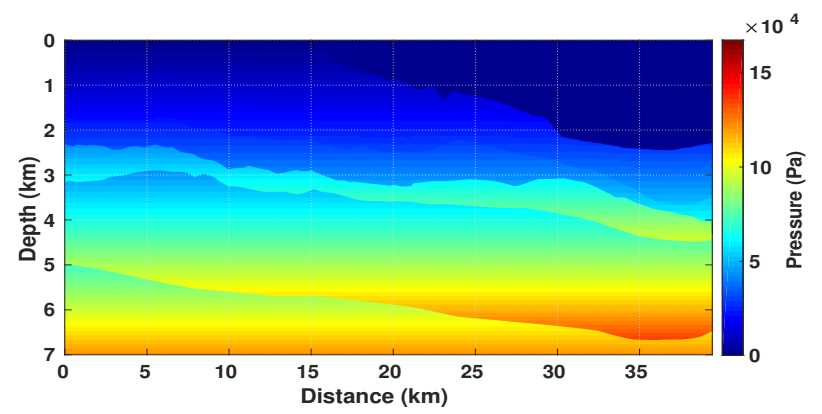

(a) Line L214-266.

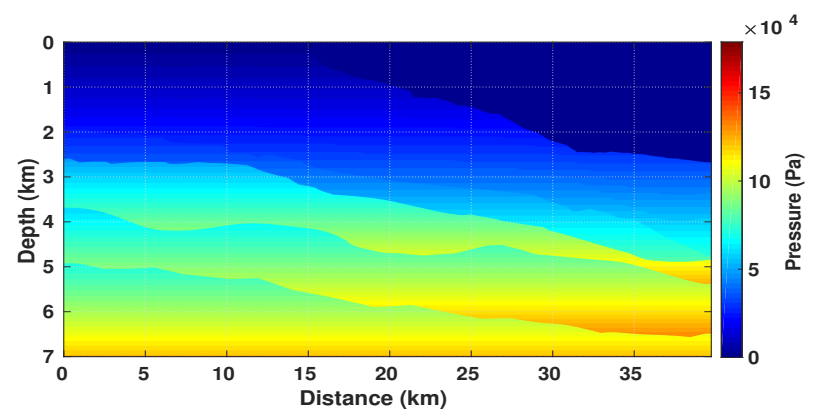

(c) Line L214-270.

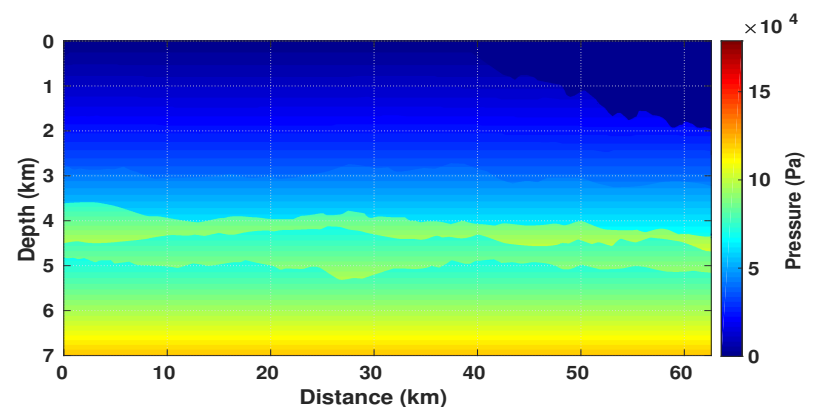

(e) Line L214-302.

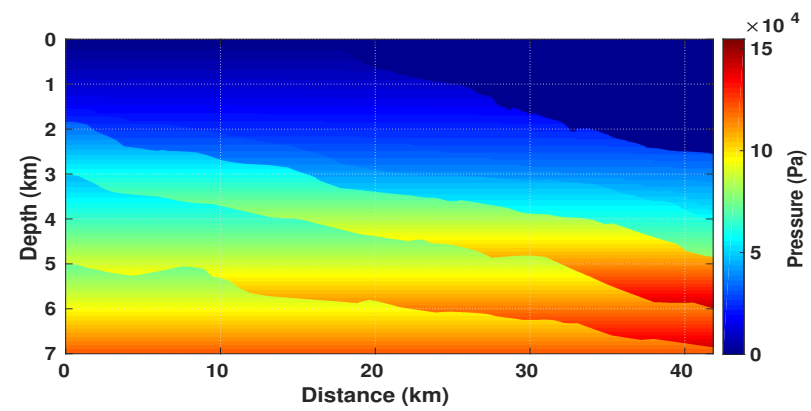

(b) Line L214-268.

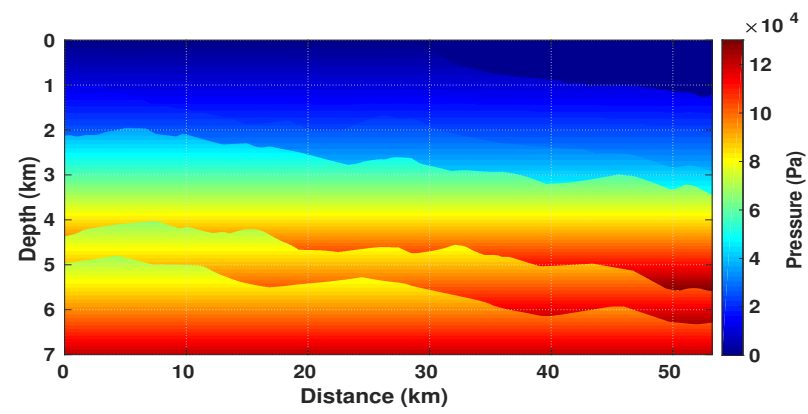

(d) Line L214-297.

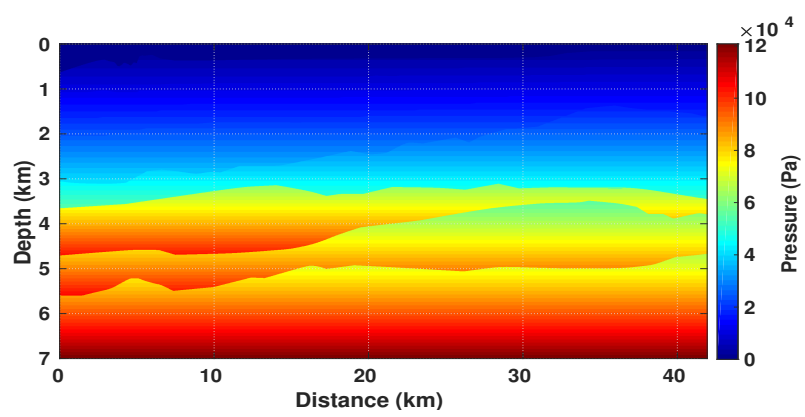

(f) Line L214-303.

Figure 12. Maps of deviatoric pressure $P_{T}(x, z)$. 


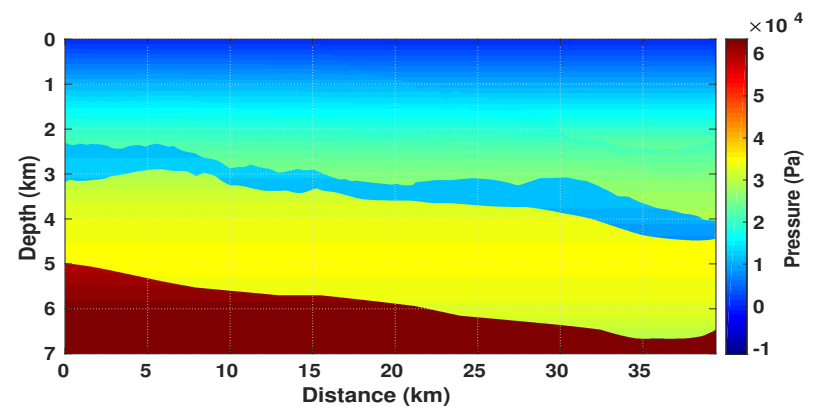

(a) Line L214-266.

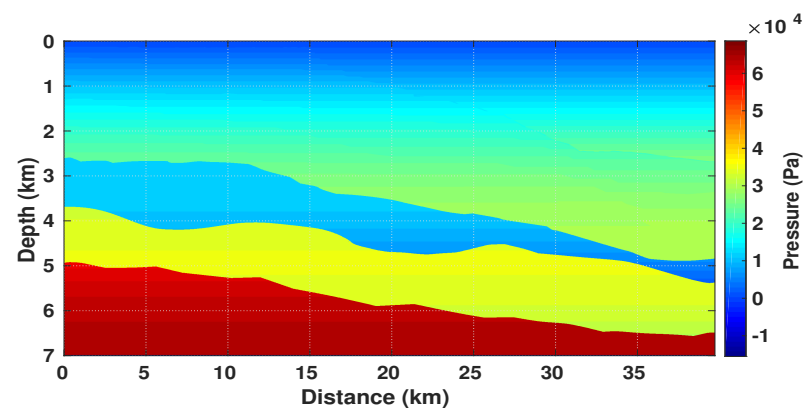

(c) Line L214-270.

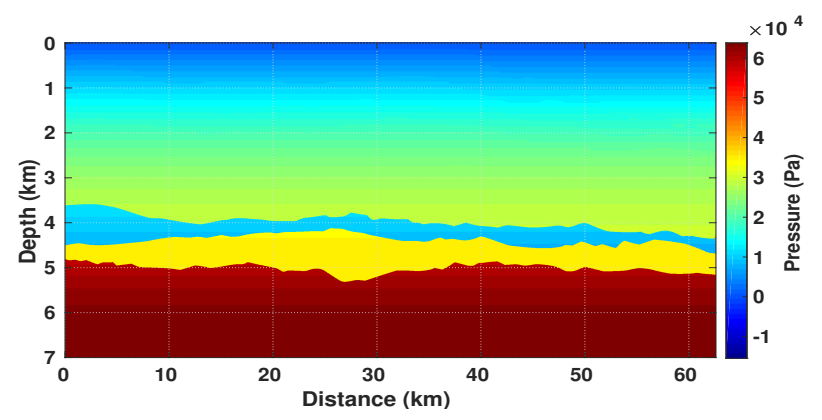

(e) Line L214-302.

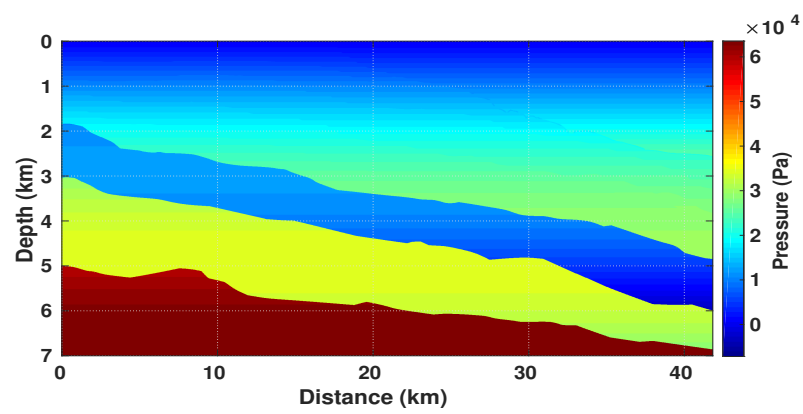

(b) Line L214-268.

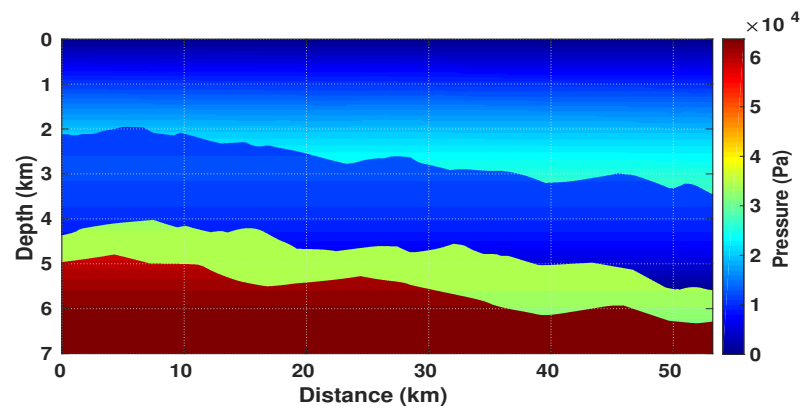

(d) Line L214-297.

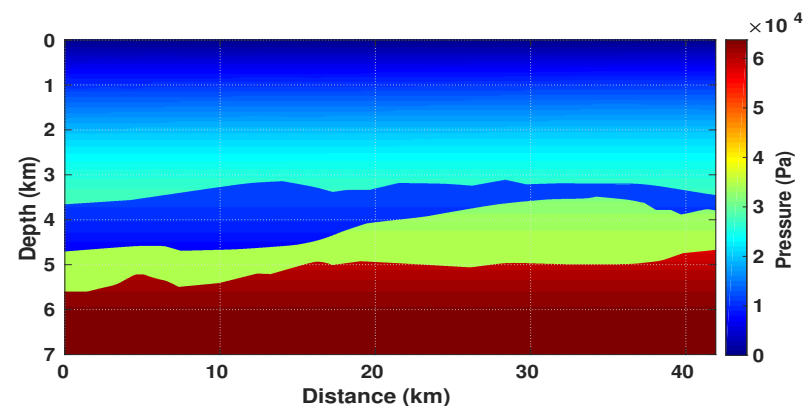

(f) Line L214-303.

Figure 13. Maps of rock pressure $P_{R}(x, z)$. 


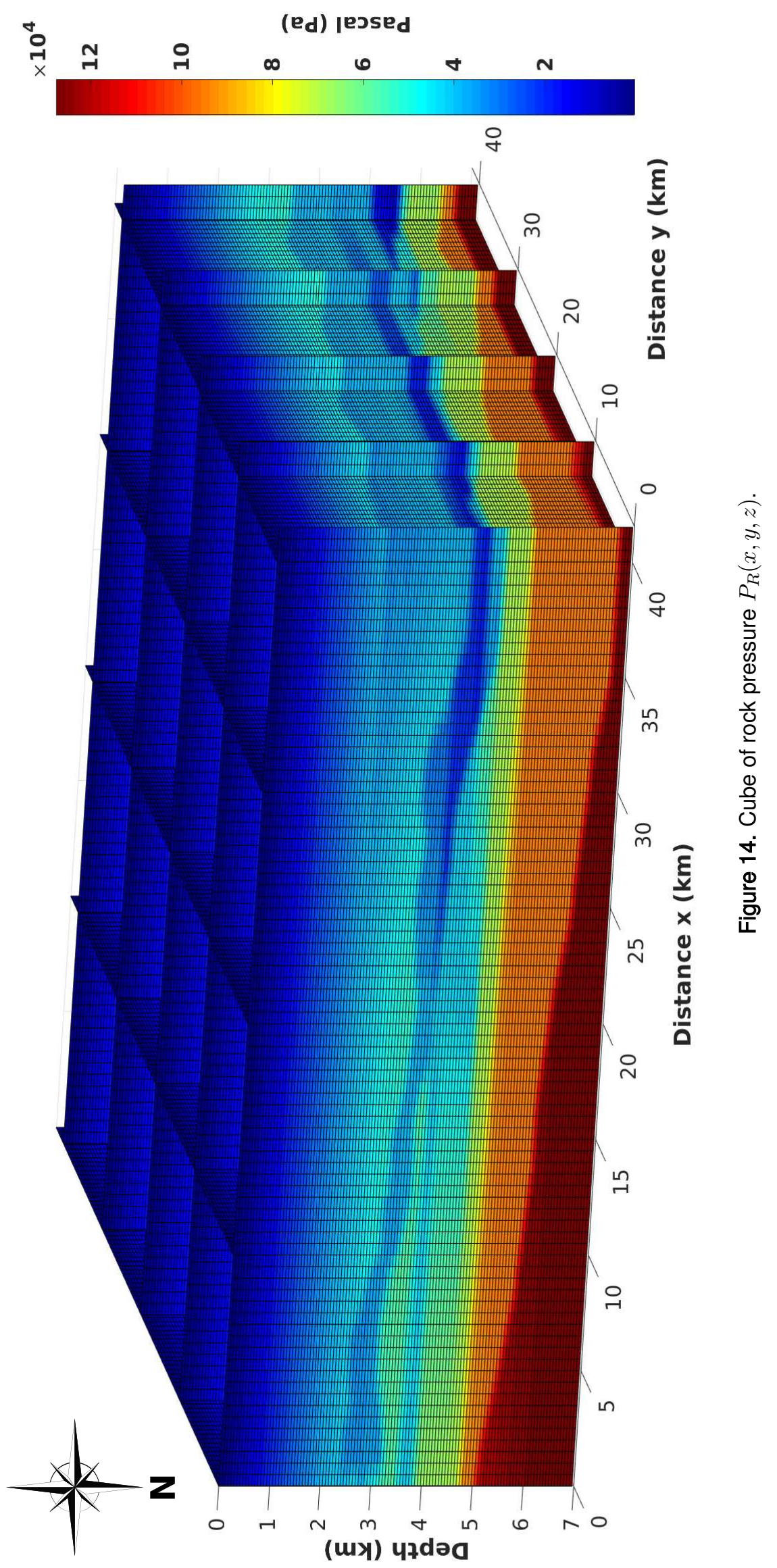

Braz. J. Geophys., 38(3), 2020 


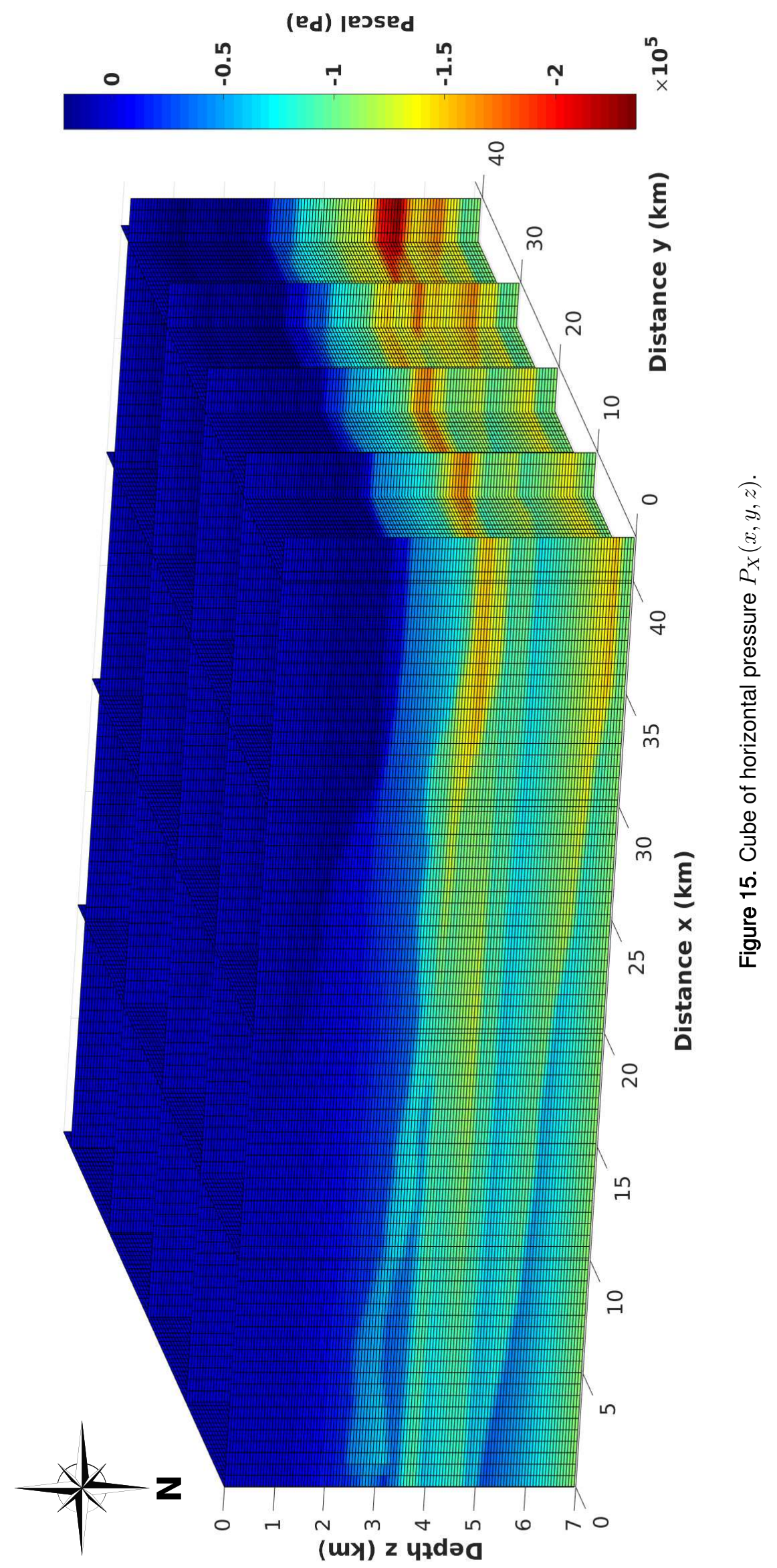

Braz. J. Geophys., 38(3), 2020 


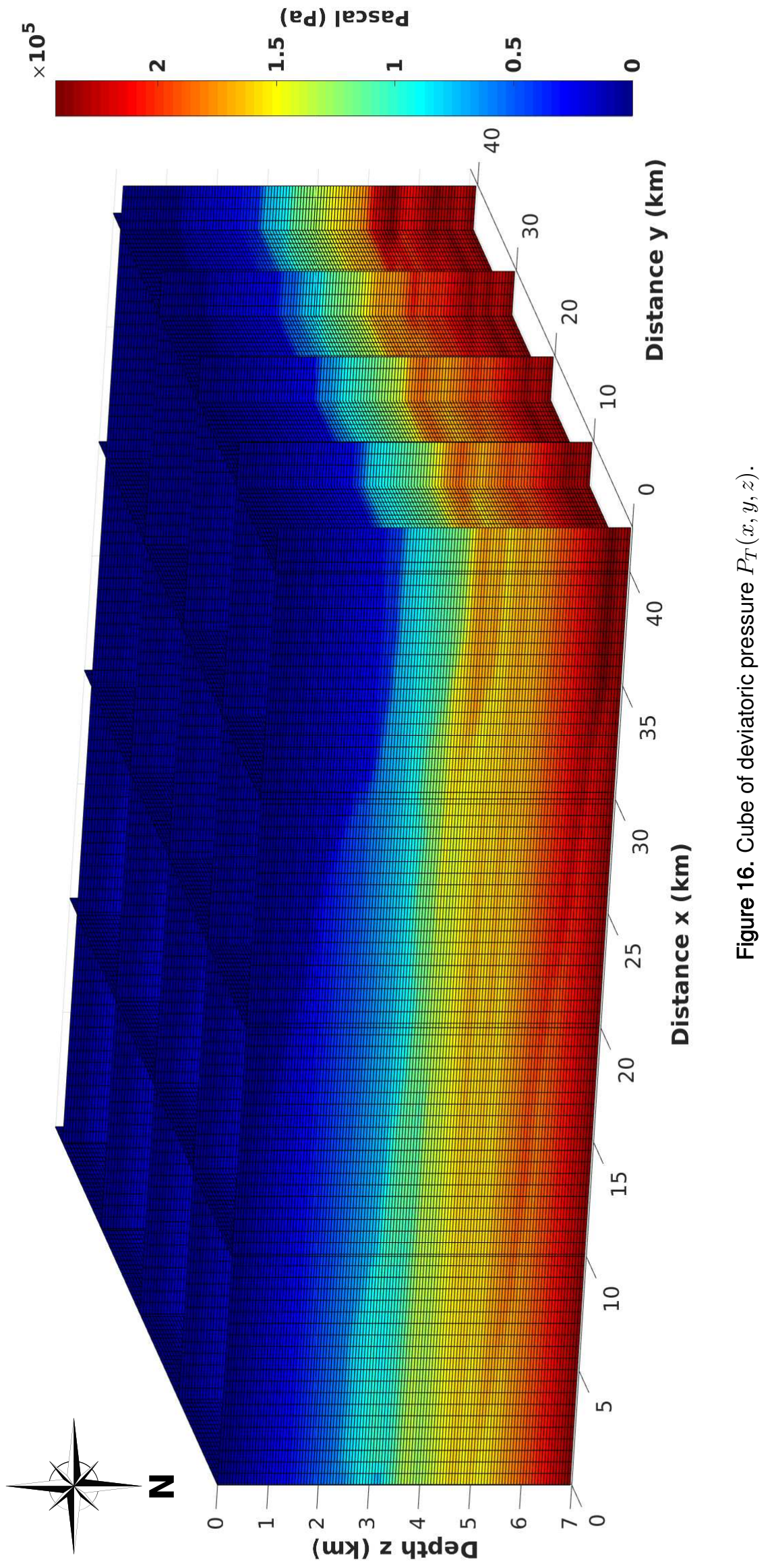

Braz. J. Geophys., 38(3), 2020 


\section{ACKNOWLEDGEMENTS}

The authors would like first to thank the reviewers for their patience and positive contributions. The authors would like to thank the Brazilian institutions UFPA (Universidade Federal do Pará), the project National Institute of Science and Technology (Instituto Nacional de Ciência e Tecnologia, INCT-GP, of MCT/CNPq/FINEP), and CENPES/PETROBRAS for the research support aiming at oil and gas exploration.

\section{REFERENCES}

ANDRADE F.T.B. 2018. Modelo 3D da Predição de Pressão para um Bloco da Bacia do Jequitinhonha. Master Dissertation on Geophysics, Instituto de Geociências, Universidade Federal do Pará, PA, Brazil. $180 \mathrm{pp}$.

COHEN J.K. \& STOCKWELL J.J.W. 2005. CWP/SU: Seismic Unix release 39 - a free package for seismic research and processing. Center for Wave Phenomena, Colorado School of Mines.

DAVIS R.O. \& SELVADURAI A.P.S. 1996. Elasticity and Geomechanics. Cambridge, United Kingdom: Cambridge University Press. $217 \mathrm{pp}$.

GARDNER G.H.F., GARDNER L.W. \& GREGORY A.R. 1974. Formation velocity and density - the diagnostic basics for stratigraphic traps. Geophysics, 39: 770780.

GREGORY A.R. 1976. Fluid saturation effect on dynamic elastic properties of sedimentary rocks. Geophysics, 41(5): 895-921.

HANTSCHEL T. \& KAUERAUF A.I. 2009. Fundamentals of basin and petroleum systems modeling. Berlin, Springer-Verlag. $476 \mathrm{pp}$.
MANN J. 2002. Extensions and applications of the common reflection surface stack method. PhD Thesis, Karlsruhe University, Karlsruhe. Germany. 165 pp.

MELLO M.R., GONÇALVES F.T.T. \& NETTO A.S.T. 1994. A successful application of the petroleum system concept in the Camamu Basin offshore Brazil. In: First Point AAPG/AMPG Research Conference "Geological aspects of Petroleum Systems", Mexico City, Mexico.

PAYTON C.E. 1977. Seismic Stratigraphy - Applications to Hydrocarbon Exploration. Tulsa, OK: American Association of Petroleum Geologists. $516 \mathrm{pp}$.

PERSEN L.N. 1975. Rock Dynamics and Geophysical Exploration. Amsterdam, Holland: Elsevier Scientific Publishing Company. 270 pp.

SIBIRYAKOV B.P., LEITE L.W.B. \& VIEIRA W.W.S. 2014. Model of the structured continuum, and the relation between specific surface area, porosity and permeability. Brazilian Journal of Geophysics, 31(4): 559-568.

SIBIRYAKOV B.P., LEITE L.W.B. \& VIEIRA W.W.S. 2015. Behavior of stresses and hydrodynamics from multicomponent seismic data. Brazilian Journal of Geophysics, 33(1): 57-70.

SIBIRYAKOV B.P., SIBIRYAKOV E.P. \& LEITE L.W.B. 2019. Dynamics of underground rocks containing fluids - Application to exploration geophysics with emphasis on oil and gas. IG, Geosciences Library, UFPA, PA, Brazil. $320 \mathrm{pp}$.

VIEIRA W.W.S., LEITE L.W.B. \& SIBIRYAKOV B.P. 2017. Modeling and pressure prediction of a block of the Jequitinhonha basin. Revista Brasileira de Geofísica, 35(3): 173-186. 